Revue des patrimoines

$21 \mid 2013$

De l'art de bâtir aux champs à la ferme moderne

\title{
La Seconde Reconstruction dans l'est des Vosges
}

Jean-Yves Henry

\section{OpenEdition}

Journals

Édition électronique

URL : http://journals.openedition.org/insitu/10443

DOI : 10.4000/insitu.10443

ISSN : 1630-7305

\section{Éditeur}

Ministère de la culture

\section{Référence électronique}

Jean-Yves Henry, «La Seconde Reconstruction dans l'est des Vosges », In Situ [En ligne], 21 | 2013,

mis en ligne le 17 juillet 2013, consulté le 20 avril 2019. URL : http://journals.openedition.org/ insitu/10443 ; DOI : 10.4000/insitu.10443

Ce document a été généré automatiquement le 20 avril 2019.

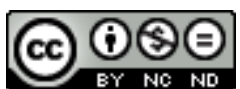

In Situ Revues des patrimoines est mis à disposition selon les termes de la licence Creative Commons Attribution - Pas d'Utilisation Commerciale - Pas de Modification 4.0 International. 


\title{
La Seconde Reconstruction dans l'est des Vosges
}

\author{
Jean-Yves Henry
}

1 L'est des Vosges ayant été particulièrement touché durant le second conflit mondial, une étude sous-thématique concernant les fermes de la Seconde Reconstruction a été développée dans le cadre d'une vaste opération consacrée à l'habitat rural de la montagne vosgienne, réalisée par le service de l'Inventaire général - Région Lorraine ${ }^{1}$. Devant l'abondance des documents conservés aux archives départementales des Vosges, une sélection s'est avérée nécessaire. Deux zones (communes de la vallée de la Meurthe - Saulcy-sur-Meurthe, Saint-Léonard et Anould - et commune de La Bresse) de tailles différentes, situées dans deux secteurs à l'habitat typologiquement différent ont été sélectionnées. La problématique a été de comprendre les règles qui ont prévalu sur le choix des partis de plans, des techniques de construction, du confort, de l'hygiène et de l'esthétique.

2 Les sources: Les archives du MRU ont été transmises par les différents services de l'Équipement qui se sont succédé, puis versées aux archives départementales des Vosges qui les conservent intégralement dans la sous-série 1152W. Celles-ci représentent plus de 100 mètres linéaires. Chaque bâtiment reconstruit fait l'objet d'un dossier constitué d'un plan de l'édifice détruit, d'un plan détaillé du bâtiment réédifié, d'un dossier comptable d'évaluation de l'indemnité et de son emploi et, le cas échéant, des courriers échangés. Les dossiers y sont classés en fonction de l'usage du bâtiment détruit : particuliers (z), industriel et commercial (DS), agricole (Ag). C'est essentiellement cette dernière catégorie de dossiers qui a été en partie dépouillée.

En novembre 1944, devant l'avancée des troupes alliées, les Allemands se retirent, dynamitant ce qui n'a pas été détruit par les bombardements des semaines précédentes. L'est du département des Vosges est particulièrement touché (fig. $\mathbf{n}^{\circ} \mathbf{1}$ ), des villages, des villes sont entièrement détruits (Saint-Dié, Gérardmer, La Bresse...). 7400 bâtiments sont sinistrés à plus de $75 \%$; parmi ceux-ci, on dénombre 3700 habitations, 2000 fermes, 1700 locaux industriels ou commerçants, entraînant 25000 sinistrés sans abri². 
Figure 1

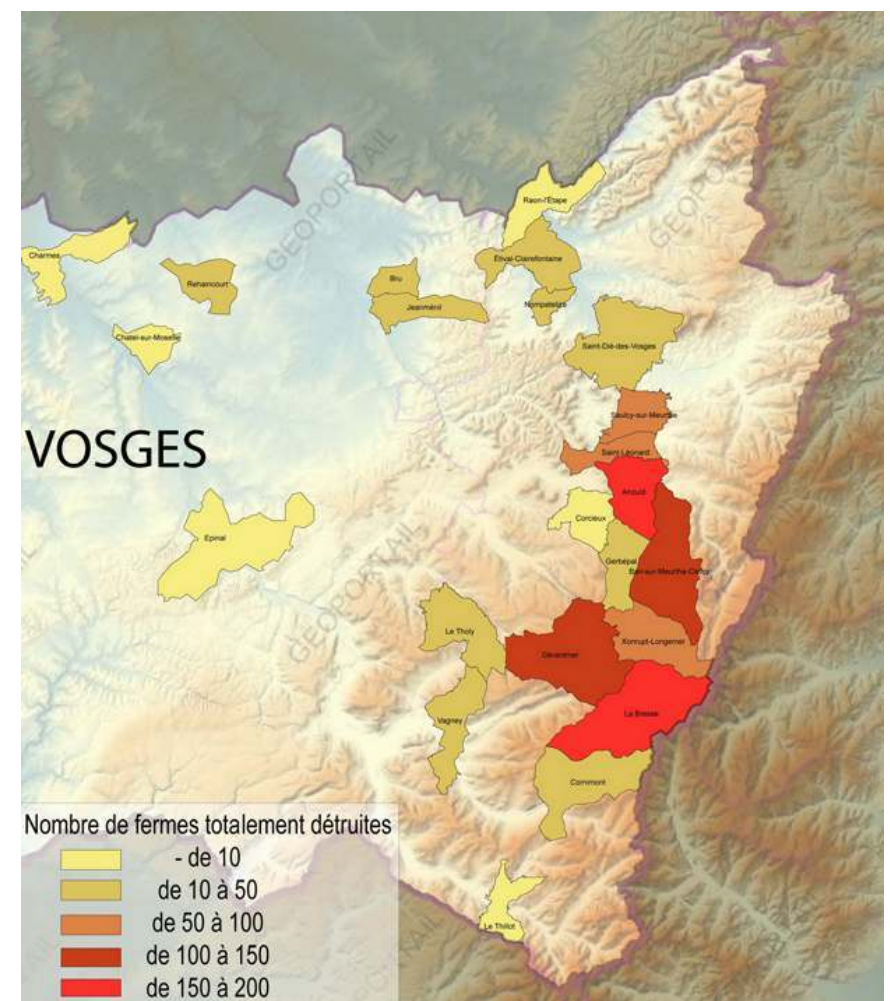

Cartographie des fermes totalement détruites par commune dans l'est des Vosges. Sources, arch. dép. des Vosges 1152W.

Cartogr. Henry, Jean-Yves. Base Mémoire : IVR41_20128812154NUDA. @ Région Lorraine-Inventaire général, 2012.

\section{L'organisation de la Reconstruction}

\section{L'organisation administrative de la Reconstruction}

Dès le 11 octobre 1940, le gouvernement de Vichy vote une loi relative à la reconstruction des immeubles détruits par fait de guerre. Il ne se contente plus comme pour la reconstruction qui suivit la Première Guerre d'en financer les indemnités, mais il crée le Comité national d'urbanisme (26 mai 1941) chargé d'en contrôler l'exécution. En novembre 1944, le gouvernement provisoire crée le ministère de la Reconstruction et de l'Urbanisme sous la direction de Raoul Dautry et la loi du 28 octobre 1946 reconnaît aux sinistrés le droit à réparation intégrale. Les dommages de guerre sont alors calculés sur la base de la valeur du bien détruit.

Chaque département est doté en 1946 d'une délégation départementale du MRU, qui approuve les plans de reconstruction et d'aménagement (PRA) et gère administrativement les opérations de reconstruction. Les départements sont divisés en arrondissements correspondant aux divisions administratives actuelles avec à leur tête un architecte en chef $^{3}$, ces derniers partagés en trois ou quatre subdivisions avec un chef de groupe qui coordonne les architectes d'opération. L'Ordre des architectes est créé en 1940, mais pour participer à la reconstruction des bâtiments publics et des travaux gérés par les ASR, tout praticien doit être agréé par le MRU4. 
6 En parallèle, l'État encourage la formation d'associations chargées de regrouper et représenter les propriétaires sinistrés. Tout d'abord dénommées Associations syndicales de remembrement, elles deviennent Associations syndicales de reconstruction (ASR) une fois celui-ci terminé. Le département des Vosges en compte quatorze, en moyenne une pour deux cantons. Leur mission est d'assurer le lien entre l'administration et les sinistrés, mais elle va bien au-delà puisque les associations participent à la définition des programmes de reconstruction, gèrent les avances de l'État et se substituent aux sinistrés dans le cadre des Opérations préfinancées (OP).

\section{L'organisation matérielle}

7 Les missions confiées au MRU sont chronologiquement de déblayer les décombres, de reloger les sinistrés, puis de reconstruire les infrastructures, l'outil de production et, dans un dernier temps, les habitations des particuliers.

Dans chaque commune sinistrée, les architectes de groupe proposent des plans de reconstruction et d'aménagement (PRA). Ceux-ci définissent les zones d'implantation des édifices publics et des équipements sportifs, les quartiers à lotir, les zones à vocation industrielle et les modifications de voierie (rectification ou/et élargissement). Des plans parcellaires délimitant les secteurs à remembrer et les zones d'égales valeurs sont ensuite élaborés. Le prix en est fixé par le Comité départemental de reconstruction. La propriété des terrains à remembrer est alors transférée à l'Association syndicale de remembrement qui procède aux échanges (avec soulte si nécessaire).

9 Parallèlement débute l'évaluation des indemnités de dommage de guerre (IDG). Les plans des bâtiments détruits sont dressés par les architectes d'opération qui ensuite en déterminent la valeur. De l'IDG seront déduits les constructions provisoires et les matériaux récupérables. Cette indemnité doit permettre au sinistré d'acheter un nouveau logement (immeuble sans affectation immédiate ou ISAI ou habitation bon marché ou HBM) ou de financer la reconstruction de l'immeuble détruit.

Le sinistré peut choisir librement son architecte, l'emplacement du bâtiment à reconstruire ${ }^{5}$ et les entreprises qu'il règle avec son IDG. Il peut aussi adhérer à une Association syndicale de reconstruction qui prendra totalement en charge la construction du bâtiment que le sinistré rachète ensuite grâce à son IDG (et, si nécessaire, une soulte) (fig. $\mathbf{n}^{\circ} 2$ ). 


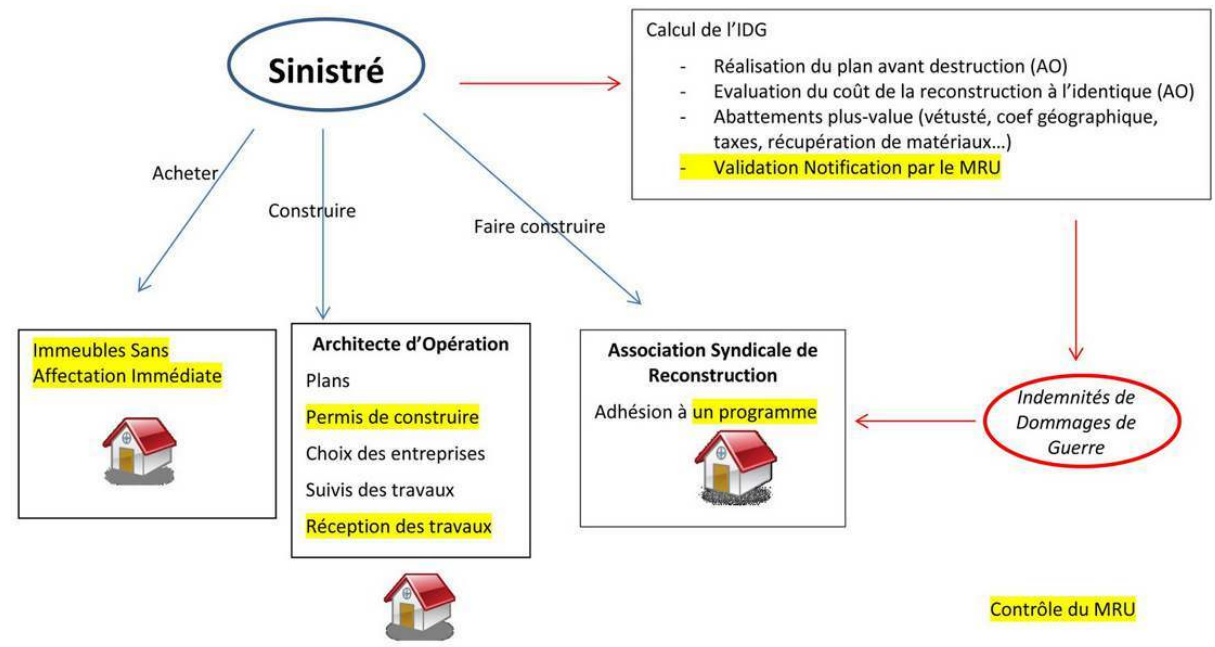

Schéma d'attribution et d'utilisation de l'indemnité de dommages de guerre.

Dessin Henry, Jean-Yves. Base Mémoire : IVR41_20128812164NUDA. (C Région Lorraine-Inventaire général, 2012.

\section{L'exemple de la commune de La Bresse}

Dans les Vosges, la première tâche du MRU est de rétablir les voies de communication et d'organiser le déblayement afin de libérer suffisamment d'espace pour y reloger provisoirement les 25000 sinistrés et y construire les camps de travailleurs.

La Bresse est vraisemblablement la commune la plus touchée par les destructions; une grande partie de l'agglomération a été détruite, mais ce sont surtout les fermes isolées qui ont été systématiquement dynamitées. Sur les 1018 bâtiments recensés en 1936, 672 sont sinistrés et 499 totalement détruits ; il faudra construire 409 bâtiments provisoires pour reloger les familles et les services.

Hormis l'église, tous les bâtiments publics sont détruits. Le PRA établi en 1945 détermine les zones industrielles (rejetées aux extrémités de la commune), les deux zones de compensation (destinées à accueillir les habitations bon marché et les immeubles sans affectation immédiate qui permettront de reloger les personnes à faible revenu ou les habitants de quartier insalubre) et les espaces de loisirs; il rectifie les axes de circulation et redéfinit l'emplacement des bâtiments publics (fig. $\mathbf{n}^{\circ} \mathbf{3}$ ). 


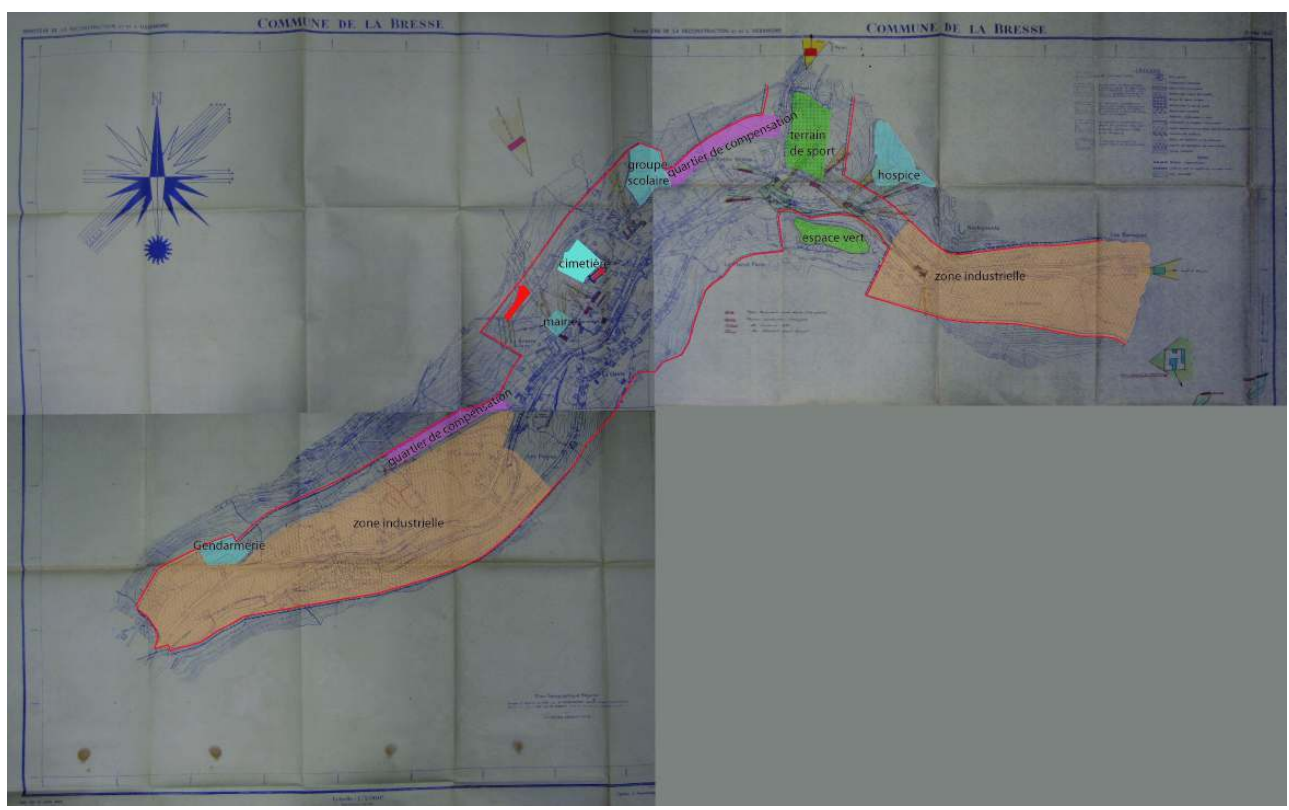

Plan de reconstruction et d'aménagement de la commune de La Bresse d'après un original. Sources, arch. dép. des Vosges 1152W810.

Dessin Henry, Jean-Yves. Base Mémoire : IVR41_20128812156NUDA. @ Région Lorraine-Inventaire général, 2012. ( Département des Vosges.

14 L'architecte urbaniste parisien Émile Deschler (1910-1991) et l'architecte d'opération vosgien Henri Gaillemin (originaire de Saulxures-sur-Moselotte) imaginent en 1946 une place centrale en pente dont le centre administratif regroupant la mairie, la poste et la salle des fêtes occupe le sommet et ferme la perspective. Les côtés de la place sont bordés par des immeubles surmontés de toitures aux faîtages décalés abritant des commerces en rez-de-chaussée. L'ensemble des constructions du centre fait l'objet d'une étude de coordination des façades (fig. $\left.\mathbf{n}^{\circ} \mathbf{4}\right)$.

Figure 4

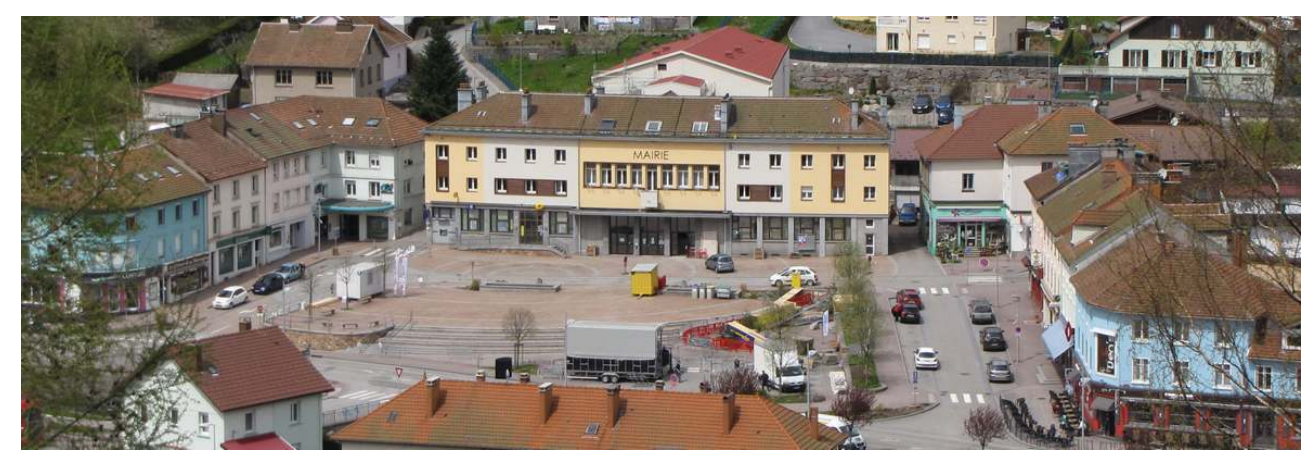

La place du Champtel à La Bresse.

Phot. Henry, Jean-Yves. Base Mémoire : IVR41_20128803335NUC2A. @ Région Lorraine-Inventaire général, 2012.

15 Parallèlement, une véritable petite ville provisoire est construite près du centre-ville sur trois terrains vierges. Elle comprend mairie, écoles, commerces et logements (fig. $\mathbf{n}^{\circ} \mathbf{5}$ ) (fig. $\mathbf{n}^{\circ} \mathbf{6}$ ). Non loin de là, le camp de l'office national pour le cantonnement des ouvriers 
de la Reconstruction (ONCOR) abrite près de 150 ouvriers ; plus loin encore, un terrain accueille le logement sous tente de l'AJAR (aide des jeunes à la Reconstruction) ; enfin, des baraquements ruraux éparpillés au loin s'élèvent à proximité des fermes détruites. Globalement, ce sont plus 420 bâtiments provisoires qui accueillent les sinistrés.

Figure 5

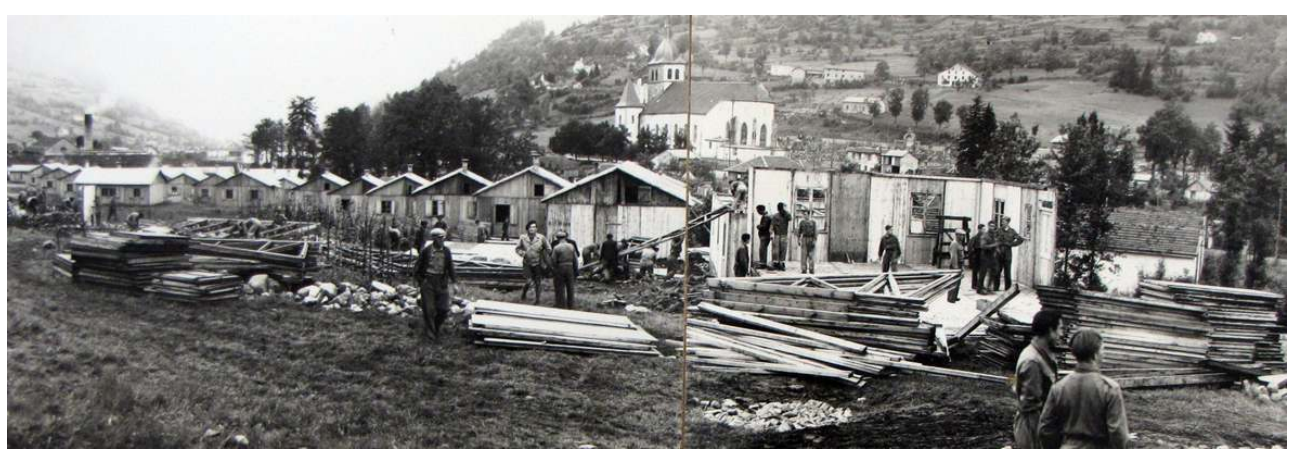

CONSTRUCTION D'HABITATIONS PROVISOIRES (ZF 64) À LA CLAIRIE, LA BRESSE PAR L'AJAR EN AOÛT 1946. PHotographie CONSERVÉE AUX ARCh. DÉP. DES VOSges SOUS LA COTE 1152W223.

Repro. Henry, Jean-Yves. Base Mémoire : IVR41_20128803336NUC2A. @ Région Lorraine-Inventaire général. @ Département des Vosges.

Figure 6

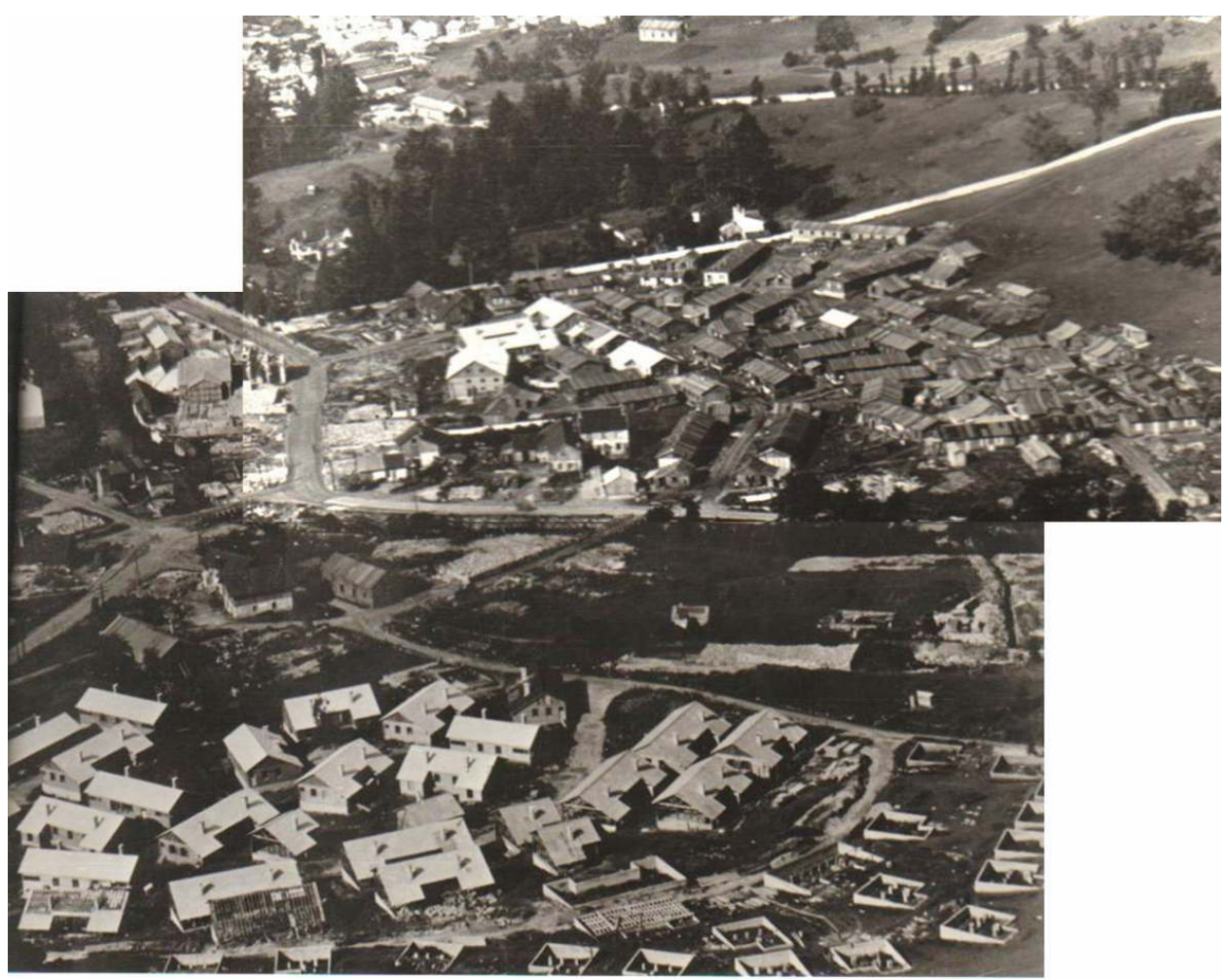

LOTISSEMENTS PROVISOIRES DU PRÉ-NOËL ET DES CHAMPIONS À LA BRESSE VERS 1950.

Collection particulière. Photomontage Henry, Jean-Yves. Base Mémoire : IVR41_20128803337NUC2A. (c) Région Lorraine-Inventaire général. 


\section{Les bâtiments ruraux}

\section{Les habitations provisoires}

Des fermes provisoires, communément dénommées «baraquements agricoles», sont rapidement érigées à proximité des bâtiments détruits, dans l'attente de la reconstruction définitive. Cet état provisoire pourra durer 10 ans dans certain cas! Un modèle unique est adopté pour tout le département des Vosges (fig. $\left.\mathbf{n}^{\circ} \mathbf{7}, \mathbf{n}^{\circ} \mathbf{8}, \mathbf{n}^{\circ} \mathbf{9}\right)$.

\section{Figure 7}

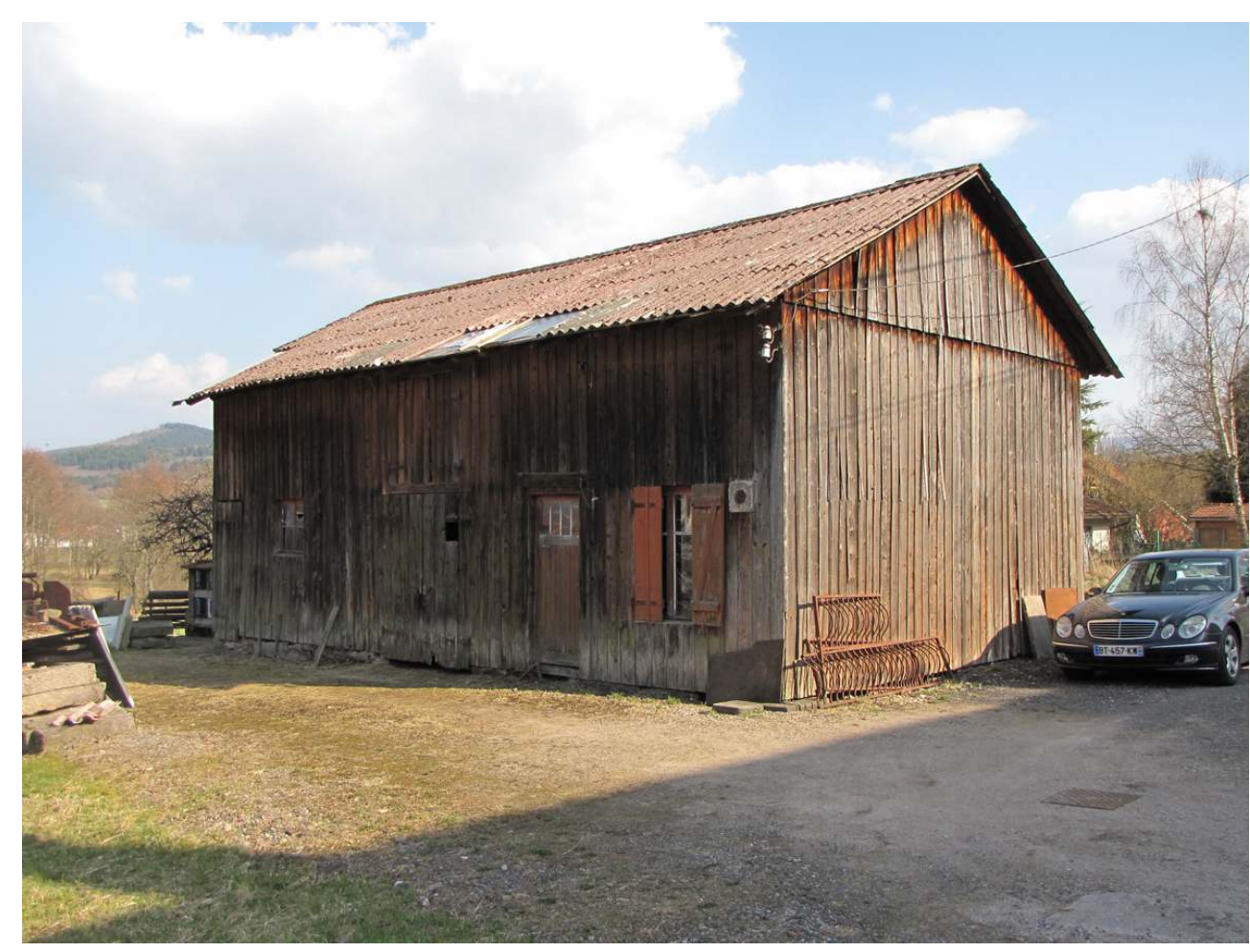

FERME PROVISOIRE À SAINT-LÉONARD.

Phot. Henry, Jean-Yves. Base Mémoire : IVR41_20128803340NUC2A. @ Région Lorraine-Inventaire général. 
Figure 8

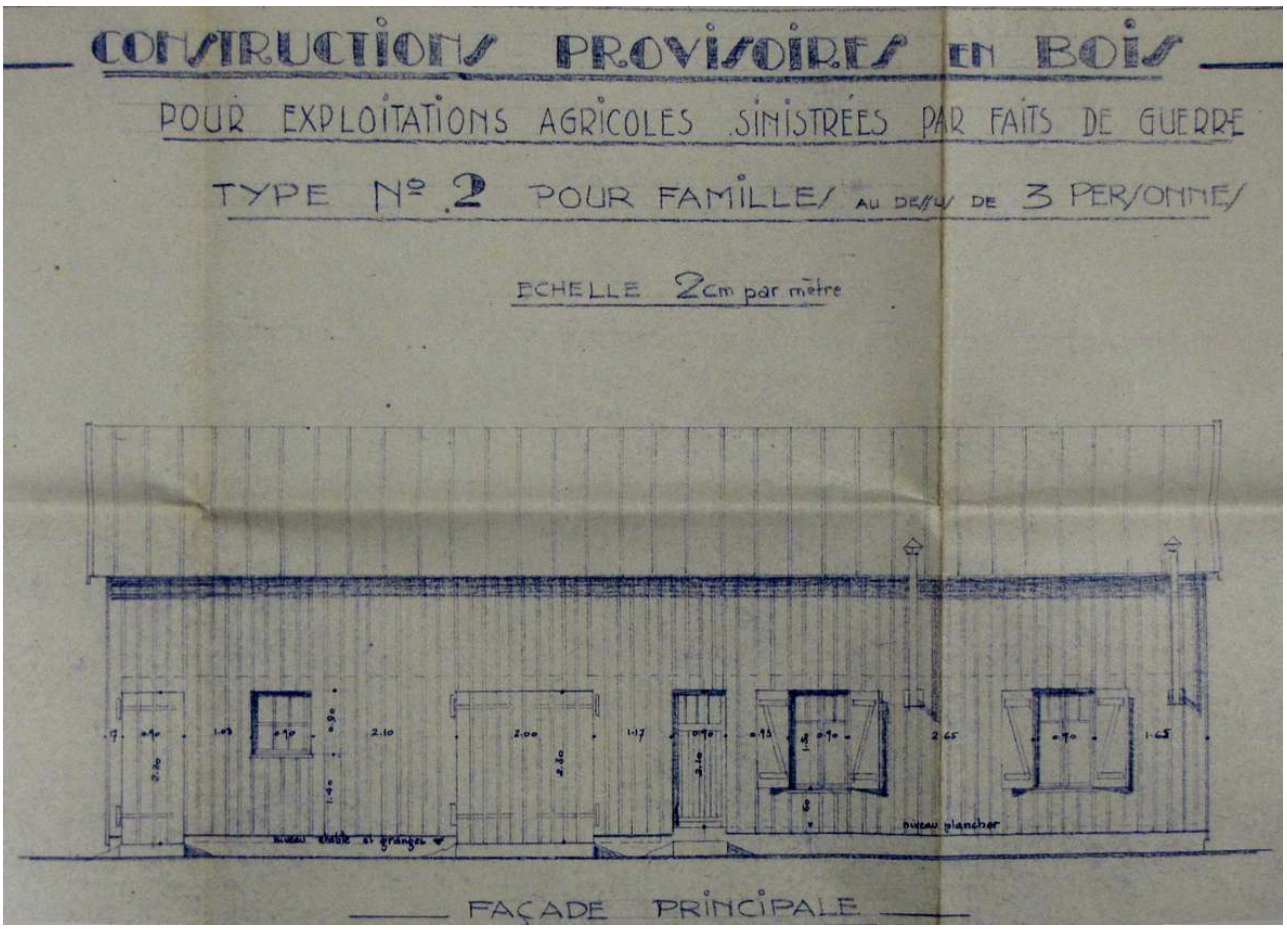

ÉLÉVATION D'UNE FERME PROVISOIRE, ARCHITECTE MARCHAL GUSTAVE, MARS 1945. DOCUMENT CONSERVÉ AUX ARCH. DÉP. DES VOSgES SOUS LA COTE EDPT77-1W.

Repro. Henry, Jean-Yves. Base Mémoire : IVR41_20128803338NUC2A. (C Région Lorraine-Inventaire général. (c) Département des Vosges.

Figure 9

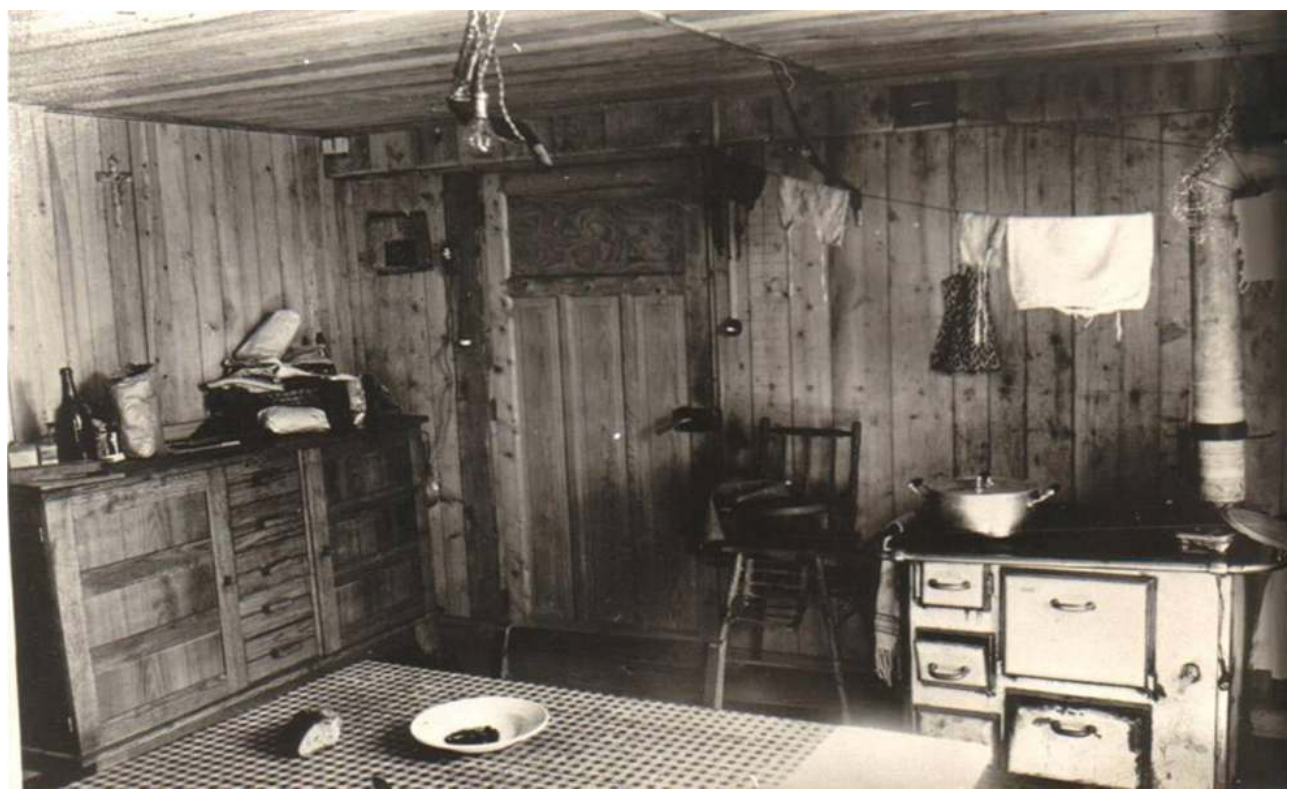

INTÉRIEUR D'UNE FERME PROVISOIRE, COLLECTION PARTICULIÈRE.

Repro. Henry, Jean-Yves. Base Mémoire : IVR41_20128803307NUC2A. @ Région Lorraine-Inventaire général. 
17 Le bâtiment à structure charpentée est construit sur des fondations maçonnées, il est fermé par des planches verticales. Le comble à surcroît est abrité par une charpente constituée de fermettes à entrait retroussé. Les parois de la partie habitation sont doublées intérieurement de papier goudronné recouvert de planches rainurées. La couverture initialement en papier goudronné sera progressivement remplacée par de la tôle ondulée ou des plaque de fibrociment.

Le logis situé en extrémité se compose d'une ou trois chambres (selon la taille de la famille) et d'une cuisine directement accessible de l'extérieur, équipée d'une cuisinière et d'un évier. L'autre extrémité est occupée par une étable pour trois bovins et animaux de basse-cour. La partie centrale accueille la grange, tandis que le comble, volume unique, abrite le fenil.

De nombreux baraquements agricoles subsistent aujourd'hui, ils ont été rachetés grâce aux indemnités et transformés en annexes agricoles.

\section{La ferme de la Seconde Reconstruction}

\section{Techniques et matériaux}

20 La Première Reconstruction avait apporté le ciment et avec lui le béton, mais ce dernier restait un matériau cher et utilisé avec parcimonie. Son emploi était limité aux linteaux de baies (béton armé), parfois aux jambages de la façade principale où, teinté, il imitait le grès rose traditionnel, prenant le nom de " pierre artificielle ». Souvent, il cédait la place à la brique silico-calcaire pour les jambages des fenêtres latérales ou aux poutrelles d'acier (profilés IPN) pour les linteaux de portes charretières. Le béton était aussi utilisé pour les sols, coulé sur une structure porteuse. Bien que connue, la technique des éléments préfabriqués ne fut pas employée.

21 Les règlements d'hygiène du début $d u X X^{e}$ siècle imposaient déjà une hauteur sous plafond de 2,50 mètres et des surfaces de baies importantes, mais ne prescrivaient rien en matière de sanitaires. Les WC des bâtiments ruraux restèrent donc localisés au fond du jardin et la salle de bain inconnue.

22 La Seconde Reconstruction n'apporte pas de nouveaux matériaux mais permet la diffusion de nouvelles techniques, la plus novatrice étant vraisemblablement la dalle sur hourdis (blocs alvéolés de béton ou de terre cuite) portés par des poutrelles de béton armé précontraintes. Les "agglos ", parpaings de béton préfabriqués, sont disponibles, mais leur emploi reste limité au mur de refend. Pour les murs extérieurs, on préfère réemployer les moellons des ruines voisines, car disponibles à volonté et surtout gratuits (fig. $\left.\mathbf{n}^{\circ} 10\right)$ ! 


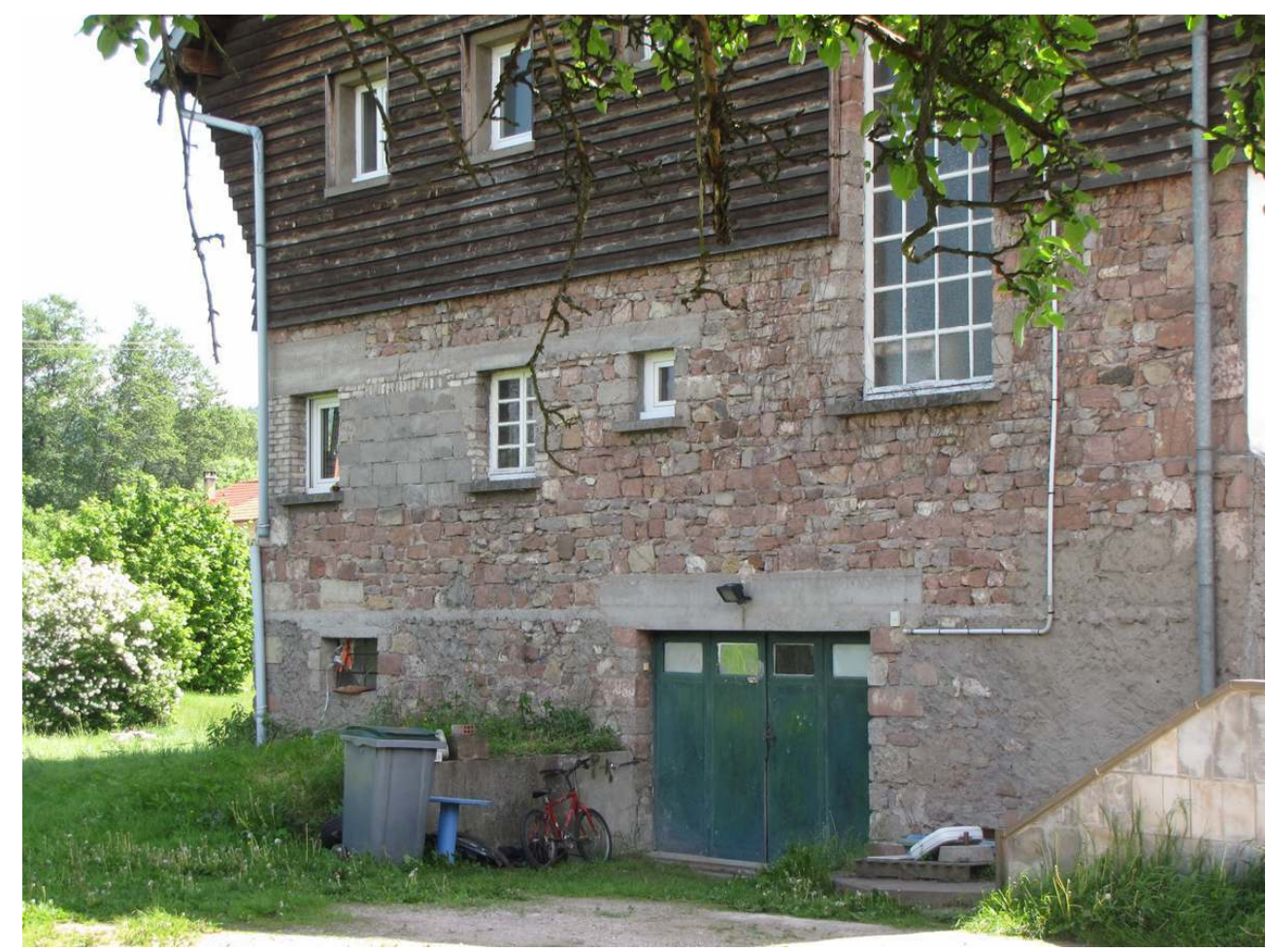

PATCHWORK DE MATÉRIAUX SUR UN BÂTIMENT RECONSTRUIT À SAINT-LÉONARD : MOELLONS DE gRÈS, PARPAINGS DE BÉTON, BRIQUES SILICO-CALCAIRE, LINTEAUX EN BÉTON ARMÉ...

Phot. Henry, Jean-Yves. Base Mémoire : IVR41_20128803308NUC2A. C Région Lorraine-Inventaire général.

\section{Hygiène et confort}

Les innovations touchent principalement le confort et l'hygiène. La hauteur sous plafond de 2,50 mètres minimum reste de mise, la surface des baies est réglementée au $1 / 10^{\mathrm{e}}$ de la surface au sol de la pièce ( $1 / 8^{e}$ dans les zones de haute montagne). Deux nouveaux espaces prennent place entre l'exploitation et le logis, la buanderie et la salle d'eau. La buanderie est, ici, un lieu destiné à la préparation des aliments pour le bétail où l'on trouve le bassin, l'emplacement du fourneau et souvent le fumoir. La salle d'eau comprend au minimum un lavabo et une douche.

24 La cuisine est séparée de la salle à manger, avec parfois un salon qui porte encore le nom de «poële " ${ }^{6}$. La partie agricole est souvent équipée d'un système de déchargement du fourrage. La charpente, toujours portée par des poteaux qui reposent sur les murs de refend, est couverte de tuiles mécaniques.

\section{Plan}

25 Jusqu'en 1952, les fermes sont reconstruites sur le plan traditionnel du bâtiment rectangulaire couvert d'un toit à deux pans, structuré en travées fonctionnelles (habitation, grange, étable et remise) perpendiculaires au faite du toit. Les surfaces au sol et les élévations à deux niveaux restent identiques, les fermes standards étant conçues pour trois à sept bovins (fig. $\mathbf{n}^{\circ} \mathbf{1 1}$ ). Après 1952, l'Opération préfinancée 24 remet en cause 
ce plan. La ferme est constituée de modules de profondeur variable, indépendants et standardisés qui s'adaptent plus précisément aux besoins des sinistrés. La composition du bâtiment donne alors à la ferme des allures variées.

Figure 11

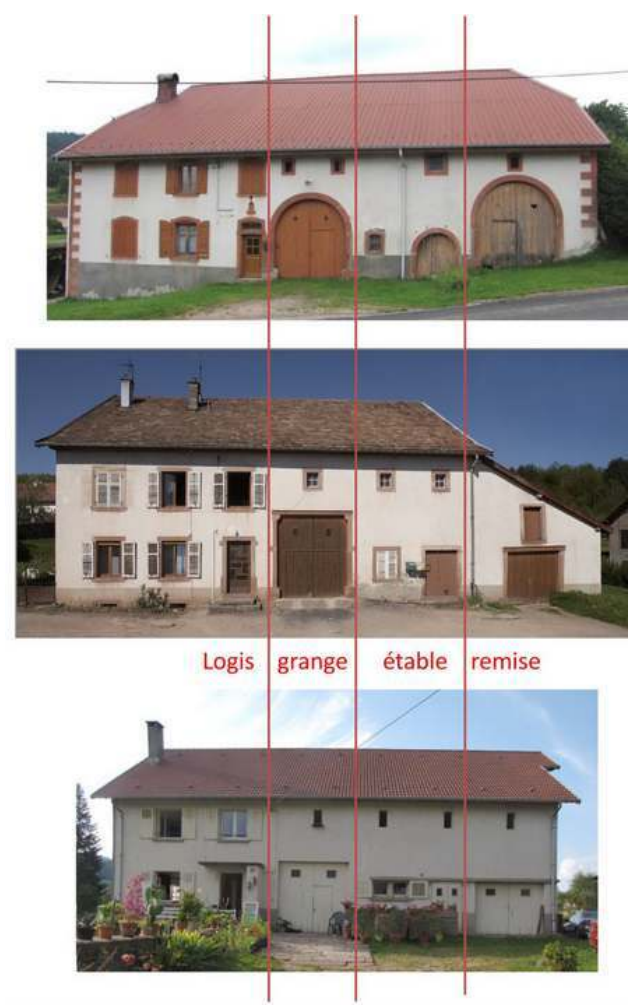

COMPOSITION EN TRAVÉES FONCTIONNELLES DES FERMES DE LA RÉgION DE SAINT-DIÉ-DES-VOSgES. DE HAUT EN BAS : FERME DU XVIIIE SIÈCLE, FERME DE LA $1^{\text {ĖE }}$ RECONSTRUCTION ET FERME DE LA $2^{\text {DE }}$ RECONSTRUCTION AVANT 1952.

Photomontage Henry, Jean-Yves. Base Mémoire : IVR41_20128803352NUC2A. @ Région LorraineInventaire général.

\section{Décor}

Seule la porte d'accès au logis fait l'objet d'un traitement particulier : encadrement en pierre ou en béton mouluré, auvent, perron... Le grès en moellons grossièrement équarris appareillés, très prisé des architectes, se retrouve en soubassement, en pan de mur de la travée principale ou encore sur les murs de clôture (fig. $\mathbf{n}^{\circ} \mathbf{1 2}$ ). Les appuis de fenêtres sont systématiquement pourvus de tablettes débordantes avec goutte d'eau. Le bois reste largement employé pour clore le fenil, les plaques de fibrociment (ou de tôle d'acier nervurées) reprennent l'usage traditionnel de l'essentage de bois pour les murs exposés à la pluie. Les débords de toit soutenus par des bras de force sont fréquents sur la façade des parties agricoles. On trouve parfois des réminiscences de décors traditionnels : niche de statue, date de construction, remploi de pierre sculptée (fig. $\mathbf{n}^{\circ}{ }^{\mathbf{1 3}}$ ). 
Figure 12

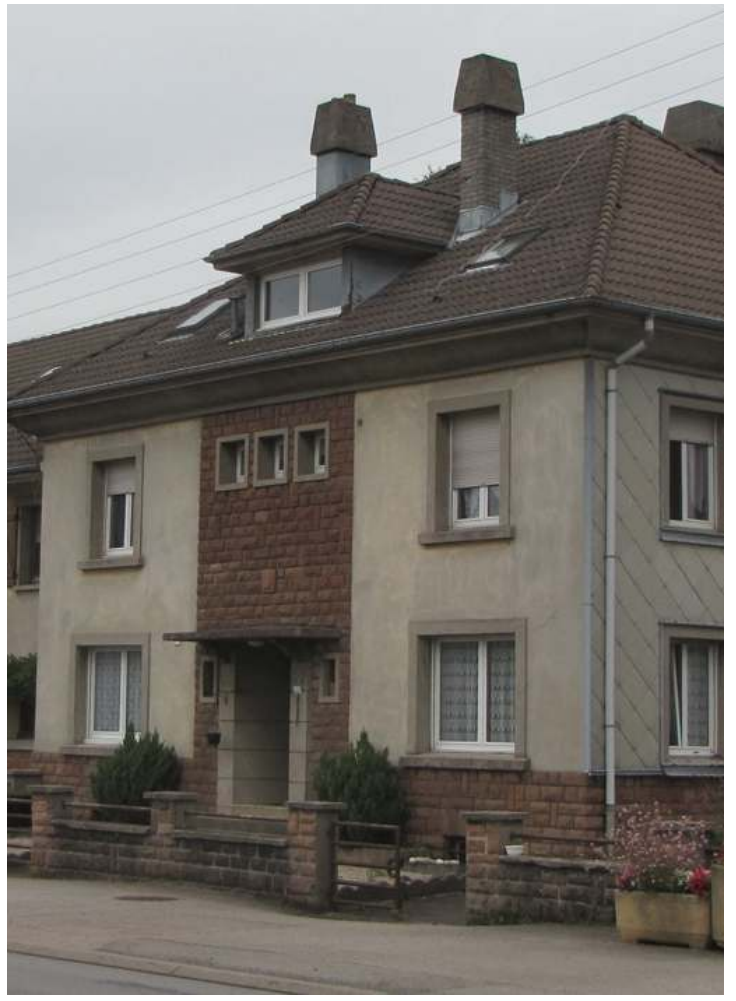

MAÇONNERIE DE MOELLONS DE gRÈS gROSSIÈREMENT ÉQUARRIS SUR LE MUR D'ENCLOS, SUR LE SOUBASSEMENT ET SUR LA TRAVÉE CENTRALE DE L'HABITATION D'UNE gROSSE FERME À SAINTLÉONARD.

Phot. Henry, Jean-Yves. Base Mémoire : IVR41_20128803306NUC2A. (C) Région Lorraine-Inventaire général. 
Figure 13

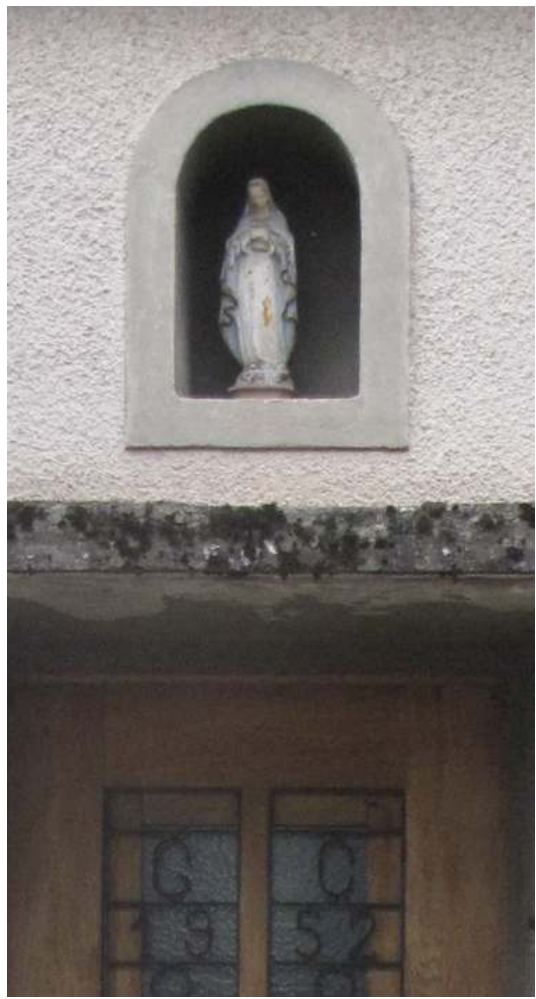

RÉINTERPRÉTATION D'UN DÉCOR TRADITIONNEL SUR LA PORTE PIÉTONNE D'UNE FERME DE SAULCYSUR-MEURTHE : NICHE ABRITANT UNE STATUETTE DE LA VIERgE, INITIALES DU PROPRIÉTAIRE ET DATE DE CONSTRUCTION DANS LA FERRONNERIE DE LA PORTE.

Phot. Henry, Jean-Yves. Base Mémoire : IVR41_20128803309NUC2A. @ Région Lorraine-Inventaire général.

\section{La ferme pilote de La Bresse}

Il s'agit de l'un des premiers bâtiments reconstruits. Les plans dressés par l'architecte Jean D. Lucius sont approuvés par les différents services du ministère de la Reconstruction et de l'Urbanisme en octobre 1946. Les travaux débutent immédiatement pour s'achever au milieu de l'année suivante, après l'interruption hivernale. Une extension de la partie agricole est construite en 1948. 


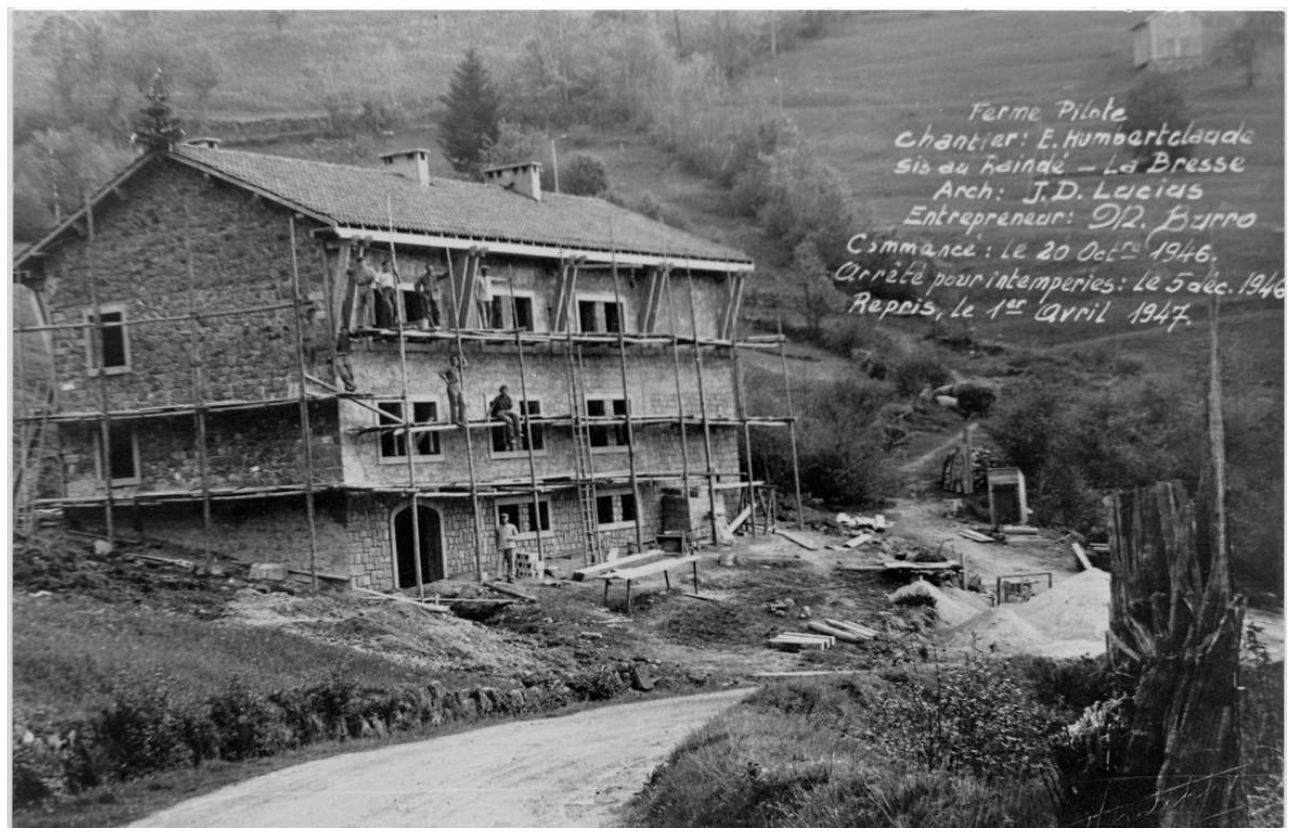

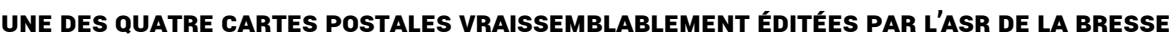
RETRAÇANT LA RECONSTRUCTION DE LA FERME PILOTE.

Collection particulière. Repro. Henry, Jean-Yves. Base Mémoire : IVR41_20128803359NUC2A. (C) Région Lorraine-Inventaire général.

La façade au soubassement en moellons apparents est largement percée de fenêtres jumelées, abritées par un avant-toit débordant soutenu par des bras de force (fig. $\left.\mathbf{n}^{\circ} \mathbf{1 4}\right)$. Le bâtiment abrite en rez-de-chaussée une fromagerie avec commerce (fig. $\mathbf{n}^{\circ} \mathbf{1 5}$ ). L'habitation se compose d'une salle commune, d'une cuisine et de WC en rez-de-chaussée et de cinq chambres et une salle d'eau à l'étage. La partie construite en retour d'équerre comporte une étable, une porcherie, un poulailler et une petite remise. En 1948, à la demande du propriétaire, le bâtiment a été prolongé d'une travée de remise surmontée d'un grenier, car, si le plan initial prévoyait une étable pour six bovins, le fenil ne pouvait accueillir que le fourrage pour trois! (fig. $\mathbf{n}^{\circ} \mathbf{1 6}$ ). 
Figure 15

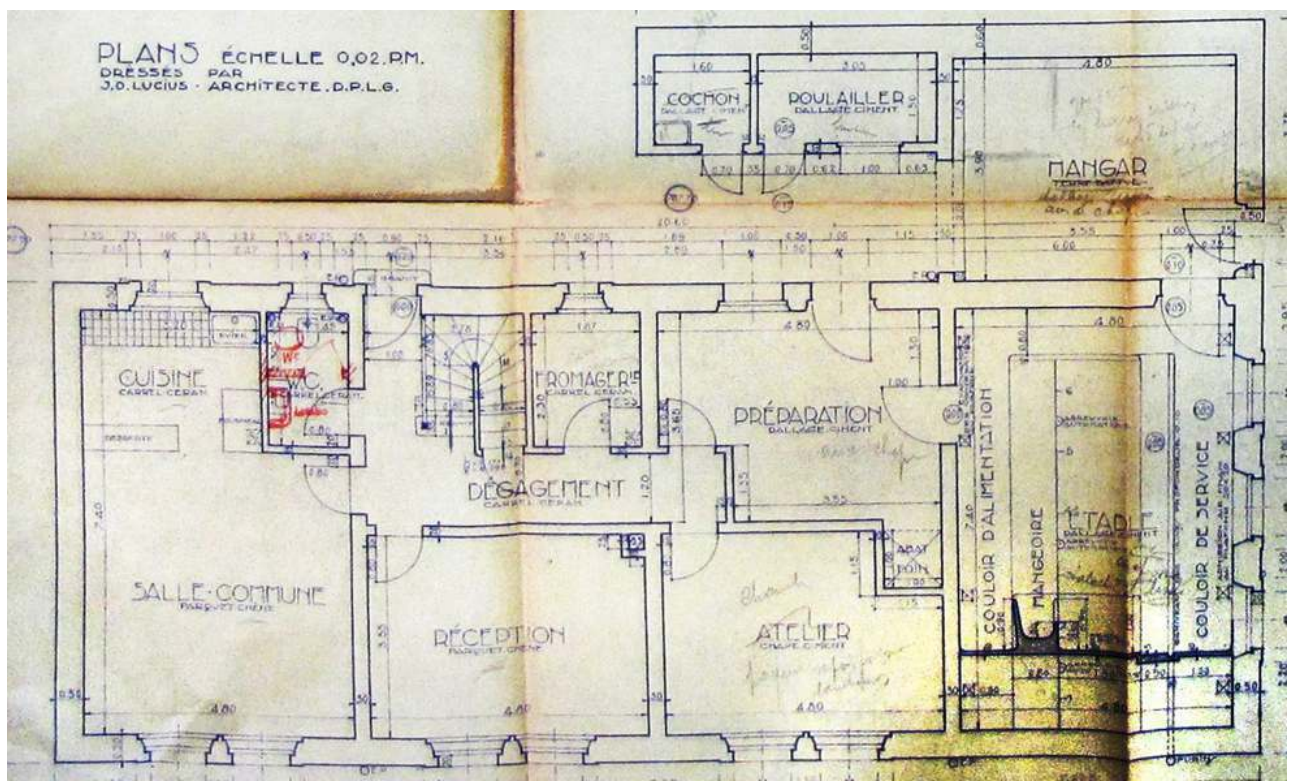

PLAN DE LA FERME PILOTE AU RAINDÉ À LA BRESSE DRESSÉ PAR L'ARCHITECTE JEAN LUCIUS EN 1946. DOCUMENT CONSERVÉ AUX ARCH. DÉP. DES VOSgES SOUS LA COTE 1152 w1380.

Repro. Henry, Jean-Yves. Base Mémoire : IVR41_20128803341NUC2A. (C) Région Lorraine-Inventaire général. (c) Département des Vosges.

Figure 16

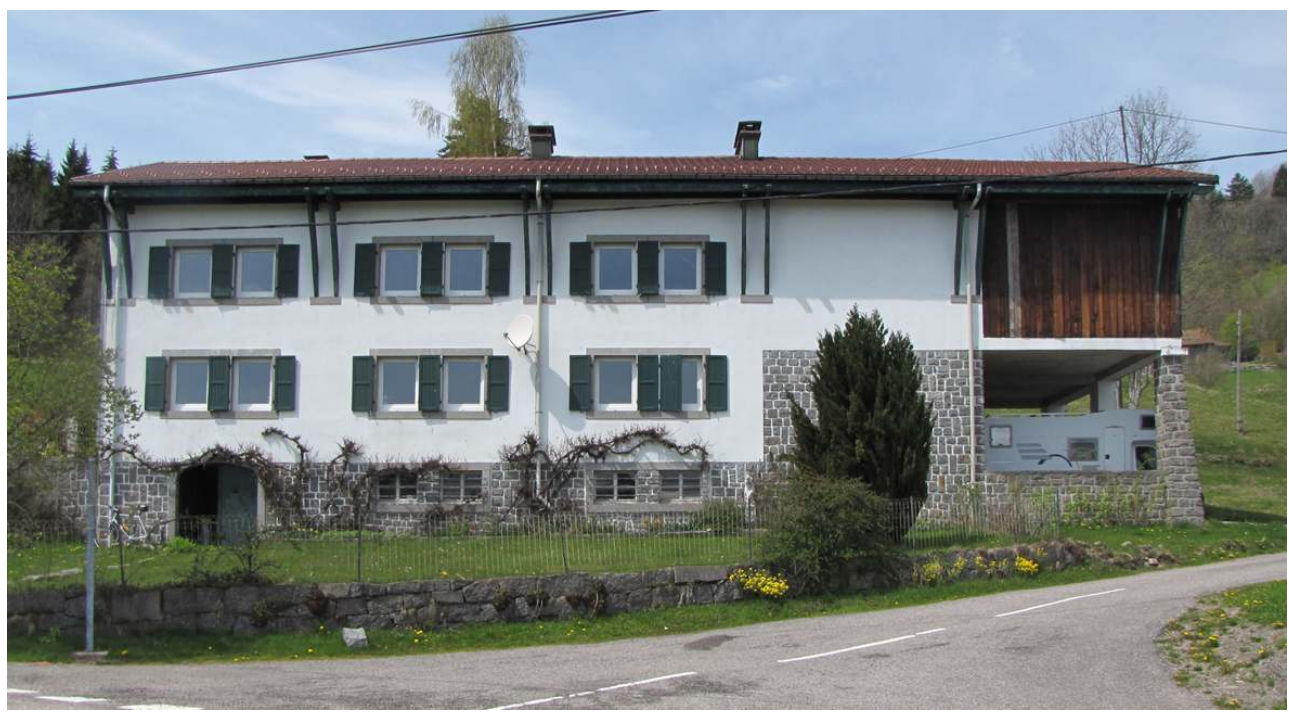

FAÇADE dE LA FERME PILOTE AU RAINDÉ À LA BRESSE.

Phot. Henry, Jean-Yves. Base Mémoire : IVR41_20128803342NUC2A. @ Région Lorraine-Inventaire général.

\section{Les Opérations préfinancées de bâtiments ruraux}

Les Opérations préfinancées (OP) sont des programmes définis par le MRU qui peuvent concerner un quartier (exemple : carrefour du centre de Saint-Léonard) ou un type de bâtiment (pavillon ou ferme) sur le territoire d'une ASR (commune ou canton). Dans les 
Vosges, huit OP ont concerné les bâtiments ruraux dits expérimentaux (OPRE) mais seulement 200 fermes ont été reconstruites dans ce cadre sur les 2000 détruites $^{7}$ (fig. $\mathbf{n}^{\circ}$ 17). Ces opérations programmées annuellement sont entièrement gérées par les ASR en fonction des avances de l'État (fig. $\left.\mathbf{n}^{\circ} \mathbf{1 8}\right)$.

Figure 17

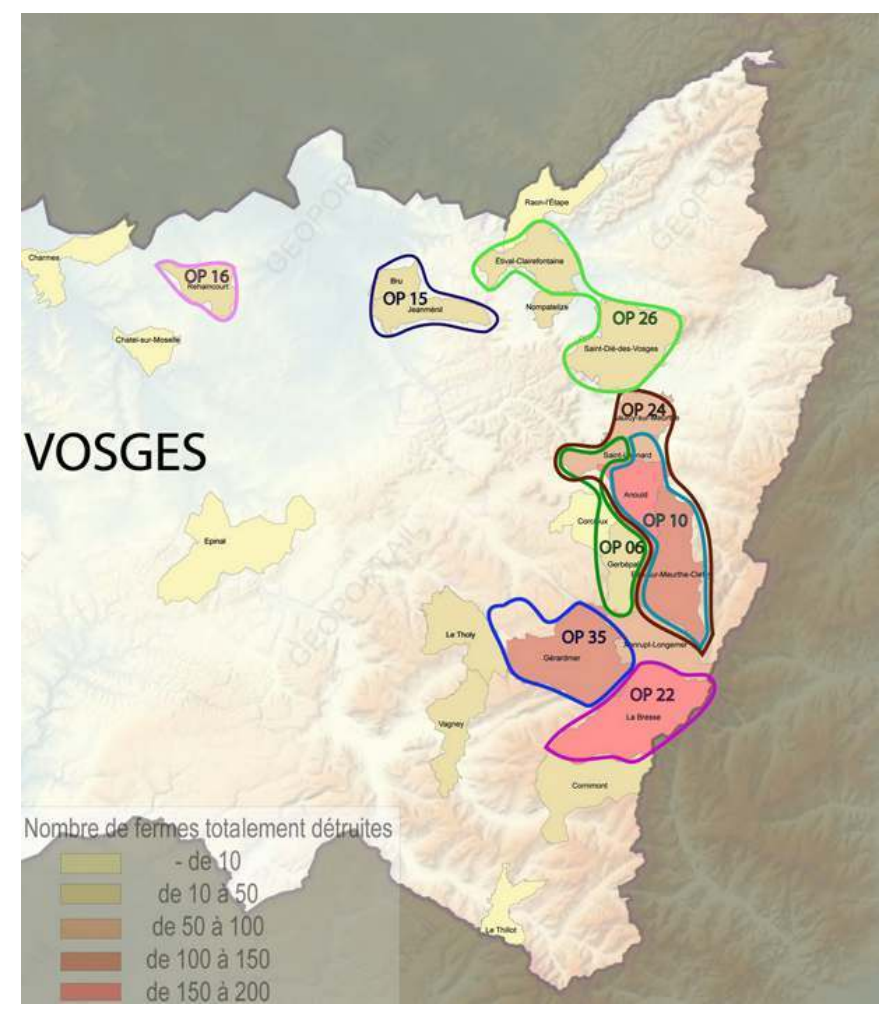

Cartographie des opérations préfinancées du département des Vosges. Sources : arch. dép. des Vosges $1152 \mathrm{~W}$

Cartogr. Henry, Jean-Yves. Base Mémoire : IVR41_20128812155NUDA. @ Région Lorraine-Inventaire général. 


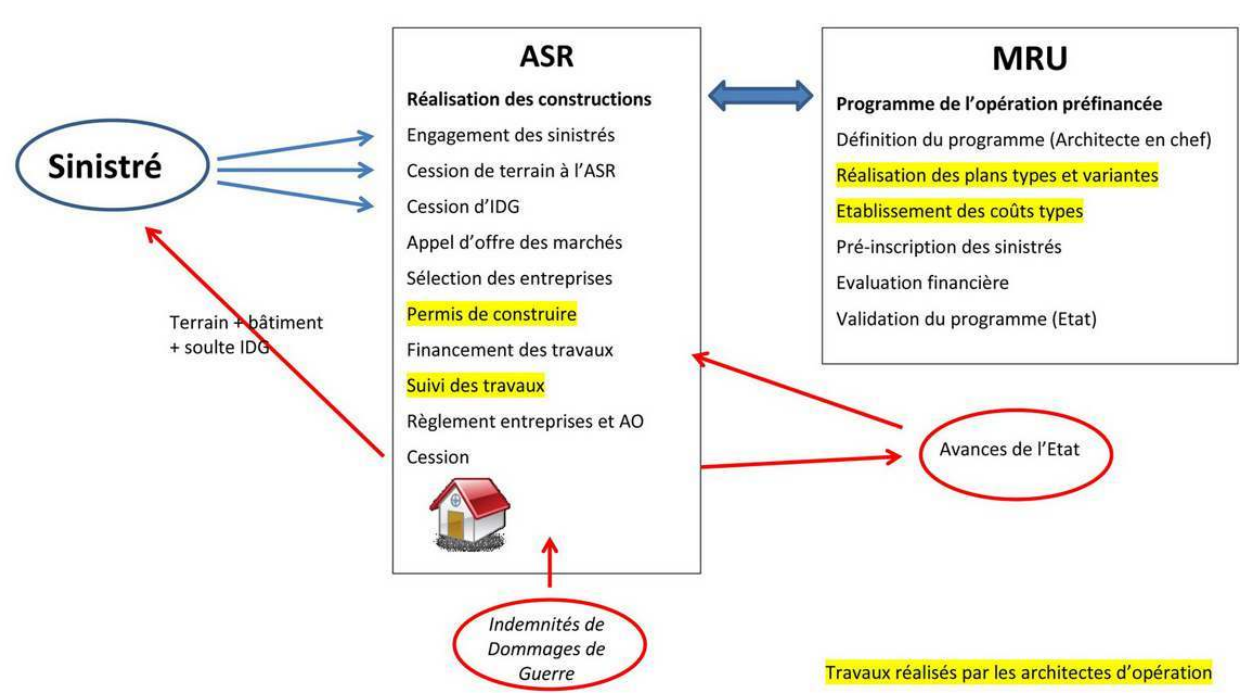

Schéma de gestion des Opérations préfinancées.

Dessin Henry, Jean-Yves. Base Mémoire : IVR41_20128812165NUDA. @ Région Lorraine-Inventaire général.

Le sinistré choisit alors le modèle et ses variantes dans un catalogue, puis il cède son terrain à l'ASR qui le lui revendra bâti, moyennant son IDG. L'adhésion à une telle opération limite les choix mais garantit une construction rapide à un coût prédéterminé.

\section{L'OP 06 à Saint-Léonard}

31 La reconstruction des fermes de la montagne vosgienne commence réellement en 1950 par l'Opération préfinancée $\mathrm{n}^{\circ} 06$ qui concerne 21 immeubles ruraux expérimentaux sur les communes de Saint-Léonard (5), Corcieux (1) et Gerbépal (15). L'opération est portée par l'Association syndicale de reconstruction de Corcieux. L'architecte Roger Ringwald de Saint-Dié propose le plan d'une ferme qui reprend la composition traditionnelle (fig. $\mathbf{n}^{\circ}$ $\left.19, n^{\circ} 20\right)$. 
Figure 19

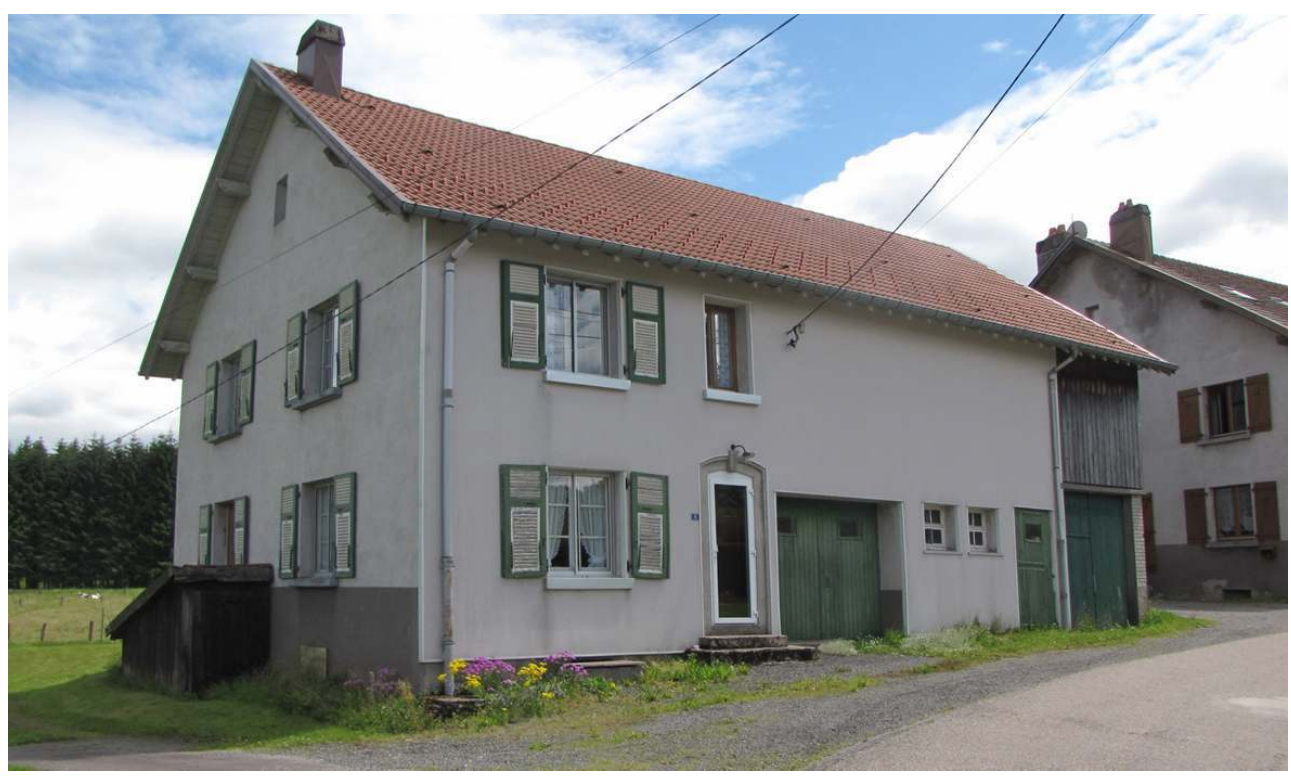

FERME DE L'OP 06 DE TYPE 2/2 À GERBÉPAL.

Phot. Henry, Jean-Yves. Base Mémoire : IVR41_20128803343NUC2A. @ Région Lorraine-Inventaire général.

Figure 20

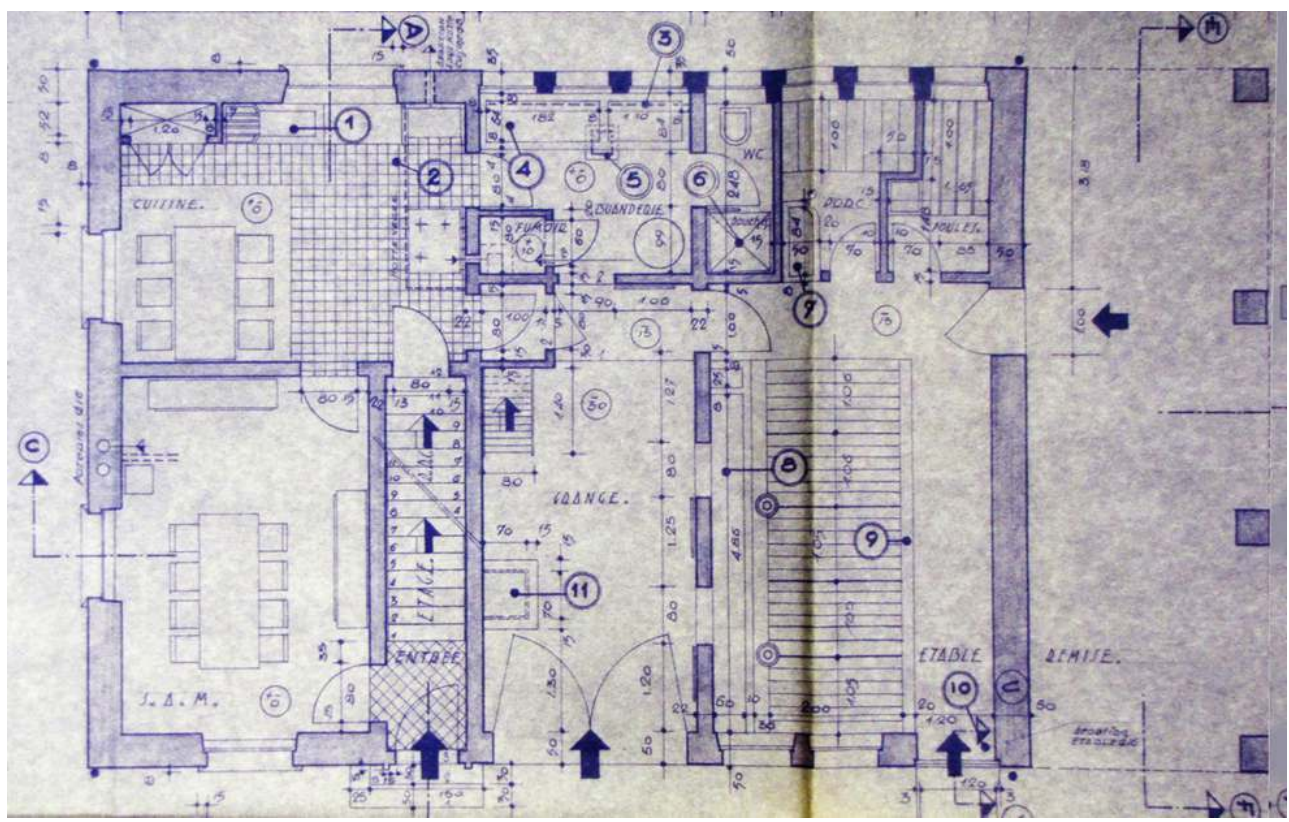

PLAN DU REZ-DE-CHAUSSÉE D'UNE FERME DE L'OP 06 DE TYPE 2/2 DRESSÉ PAR L'ARCHITECTE ROgER RINGWALD EN 1950. DOCUMENT CONSERVÉ AUX ARCHIVES DÉPARTEMENTALES DES VOSgES SOUS LA COTE 1152 w683.

Repro. Henry, Jean-Yves. Base Mémoire : IVR41_20128803344NUC2A. @ Région Lorraine-Inventaire général. @ Département des Vosges.

Le bâtiment est structuré en travées fonctionnelles dont la profondeur est adaptée à celle du logis. Il se décline en deux versions de profondeur différente : le « $2 / 2$ » dont le logis compte deux pièces et le « $3 / 3 »$ qui comprend en plus un salon. Le logis de la version 
« $2 / 2$ » se compose d'une cuisine, d'une salle à manger et de deux chambres à l'étage. La buanderie se situe au fond de la travée d'engrangement ; la travée d'étable, conçue pour cinq bovins, abrite une porcherie et un poulailler, les WC et la douche étant relégués au fond de celle-ci. Une travée de remise ouverte (optionnelle) permet de ranger et de décharger les voitures. L'étage est essentiellement occupé par le fenil et le grenier. Le logis de la version « $3 / 3$ » comporte un salon et une chambre supplémentaire à l'étage, l'étable étant prévue pour sept bovins. Pour des raisons de sécurité anti-incendie, le logis est isolé de l'exploitation par des murs coupe-feu et par un plancher de comble en hourdis de terre cuite sur poutrelles en béton armé. Le logis est équipé de WC, d'une douche et d'un chauffe-eau au gaz, la cuisine étant dotée d'un plan de travail carrelé. Dans l'étable se trouvent des mangeoires en béton et des abreuvoirs à niveau constant pour le bétail. La charpente traditionnelle est adaptée pour laisser la place à une griffe de déchargement $d u$ fourrage. Les murs extérieurs sont construits en moellons liés et enduits par un mortier bâtard de chaux hydraulique et de ciment, les murs de refend sont constitués de parpaing de béton et les cloisons de brique de terre cuite. Le béton armé est utilisé pour les encadrements de baies, ainsi que pour couvrir la cave et la travée de l'étable (poutrelles précontraintes et hourdis). La partie haute de l'exploitation est fermée par des planches verticales. Le bâtiment est couvert d'un toit à deux pans en tuiles mécaniques. Seule la porte piétonne présente un encadrement mouluré, à linteau en arc segmentaire à fausse clé en béton moulé.

\section{Les OP 22 et 22 bis à La Bresse}

L'opération concerne la reconstruction de 30 fermes sur deux années (1953-1954 et 1954-1955). Les plans sont dressés par les architectes Gaillemin et Mathieu. Ces derniers se contentent de reprendre le plan traditionnel de la ferme vosgienne de montagne en l'adaptant aux règles d'hygiène en vigueur.

Le bâtiment reprend les archétypes de la ferme locale traditionnelle: logis de deux niveaux placé du côté du pignon orienté vers la vallée, exploitation sur le pignon tourné vers la montagne. L'espace central, réduit, n'accueille encore qu'une petite grange, tandis que la rampe sur le pignon arrière donne toujours directement accès au fenil. Mais extérieurement cet édifice s'en démarque par un pan de toit largement débordant soutenu par des bras de force au-dessus des portes piétonne et animalière. La construction est déclinée en deux versions : le modèle 1 au logis de trois pièces par niveau pourvu d'une exploitation pour trois bovins et le modèle 3 dont le mur pignon, plus large, couvert d'un toit dissymétrique, accueille en plus un atelier et une étable pour cinq bovins. Le logis du modèle 1 comporte en rez-de-chaussée une cuisine, une salle commune et une chambre. L'espace traditionnellement affecté au "charri $»^{8}$ en reprend les fonctions principales : entrée et buanderie auxquelles viennent s'ajouter les sanitaires (WC et douche). L'étage est occupé par trois chambres, un cabinet de toilette, le grenier à bois et le fenil (fig. $\mathbf{n}^{\circ} \mathbf{2 1}, \mathbf{n}^{\circ} \mathbf{2 2}$ ). 
Figure 21

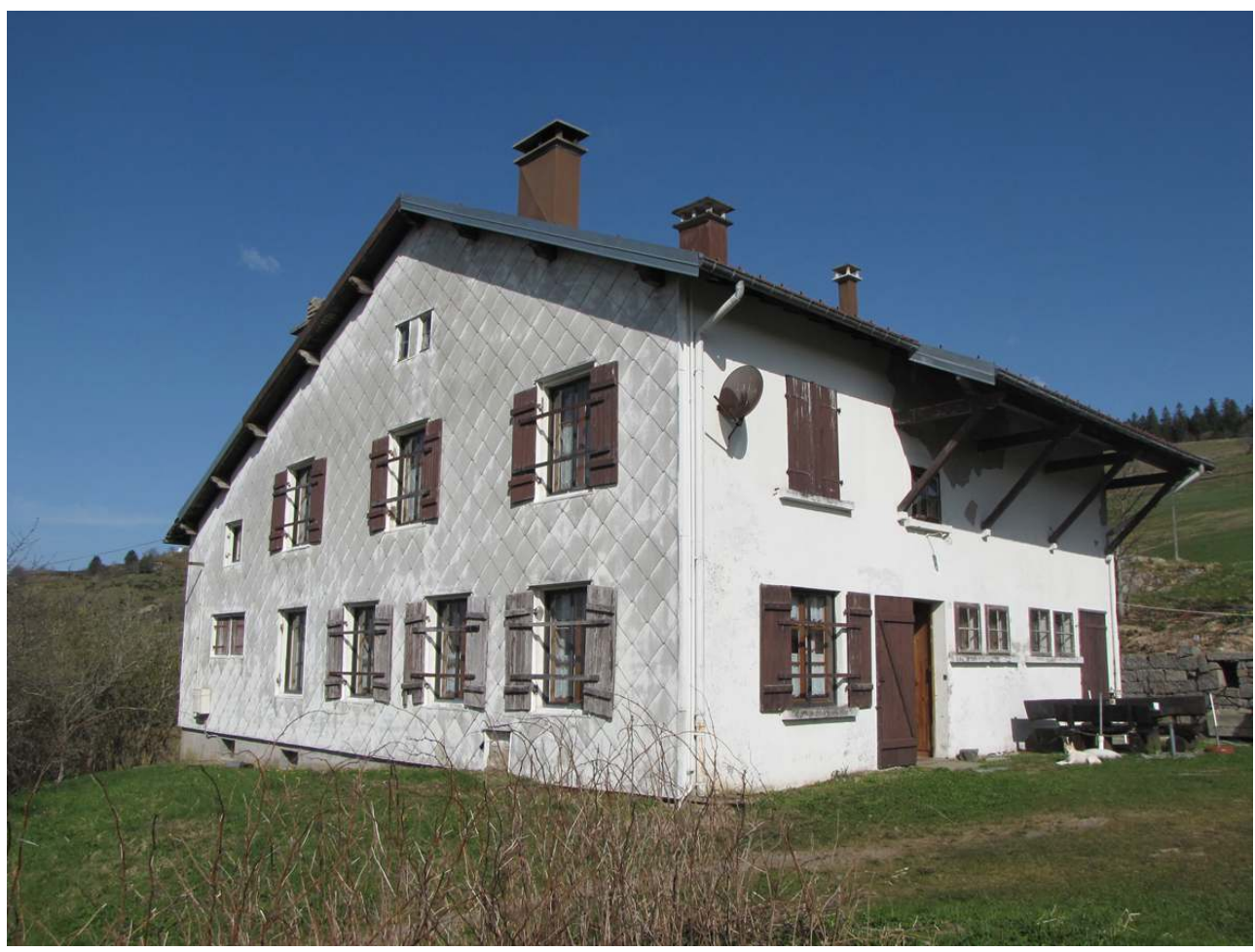

FERME DE L'OP 22 DE TYPE 3 À LA BRESSE.

Phot. Henry, Jean-Yves. Base Mémoire : IVR41_20128803345NUC2A. @ Région Lorraine-Inventaire général. 


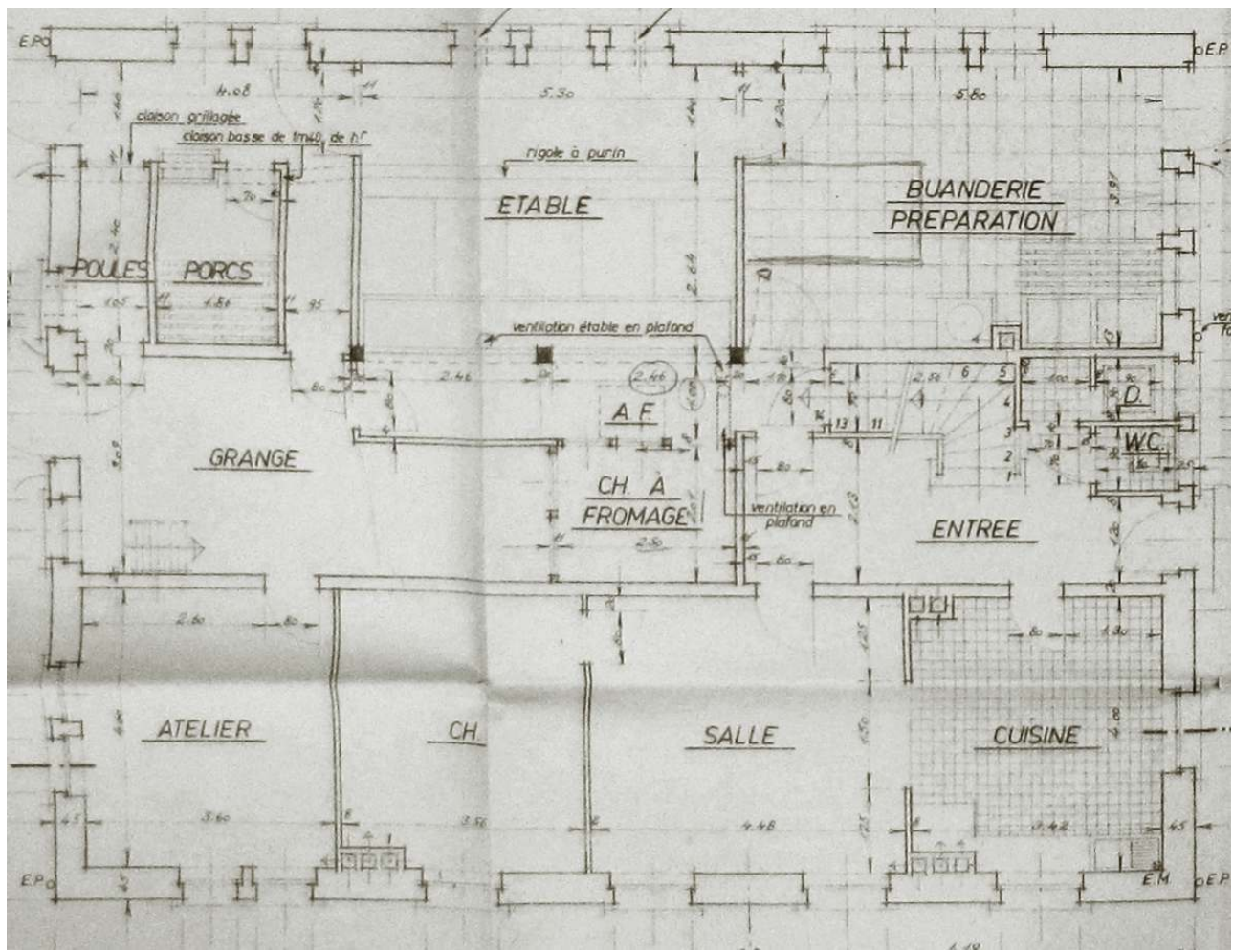

PLAN DU REZ-DE-CHAUSSÉE d'UNE FERME DE L'OP 22 DE TYPE 3 DRESSÉ PAR LES ARCHITECTES HENRI GAILLEMAIN ET LOUIS MATHIEU EN 1952. DOCUMENT CONSERVÉ AUX ARCHIVES DÉPARTEMENTALES DES VOSgES SOUS LA COTE 1152 w713.

Repro. Henry, Jean-Yves. (C) Région Lorraine-Inventaire général. Base Mémoire IVR41_20128803346NUC2A. ( Département des Vosges.

\section{L'OP 24 dans la vallée de la Meurthe}

Elle intervient après une nouvelle réflexion sur la reconstruction rurale. Celle-ci commence en octobre 1952 par le $4^{e}$ Congrès national de l'habitat rural réuni à Strasbourg et dont les conclusions insistent sur la nécessité de traiter séparément habitation et exploitation, tant sur le plan des dimensions que des matériaux, afin de mieux les adapter aux besoins des agriculteurs; de plus, la partie exploitation doit être construite comme un bâtiment industriel pour en réduire le coût et augmenter sa polyvalence. Les recommandations préconisent que le bâtiment soit équipé au mieux afin de pallier le manque de main-d'œuvre agricole.

À la suite de ce congrès, le MRU commandite une étude comparative des différentes expérimentations en France qui conclut que les OPRE des Vosges sont d'un coût de $7 \%$ inférieur à la moyenne nationale, économie due uniquement au groupement des constructions et à leur standardisation".

Le rapport du MRU des Vosges du 4 février 1953 préconise donc de séparer habitation et exploitation tout en les reliant par un corps de passage à fonction de buanderie et de lieu de préparation des aliments, d'employer les techniques d'ossature en béton armé, métal ou bois pour la partie exploitation, d'adapter la profondeur aux besoins du sinistré et d'augmenter l'équipement en appareils ménagers (cuisinière avec production d'eau 
chaude, sanitaires, machine à laver...), ainsi qu'en installations (abreuvoirs automatiques, prise d'eau pour le lavage...).

Le MRU mobilise l'ensemble les architectes agréés du secteur pour répondre à sa demande et dans la note de service du 13 mai 1953, désigne les architectes: Lucien Toussaint et Michel Trefcon pour la partie habitation, Francis Massé pour l'exploitation, Roger Ringwald et Roger Perrin pour les devis ; les architectes d'opération sont Ringwald, Perrin, Toussaint-Trefcon, Georges Michau étant architecte en chef.

Figure 23

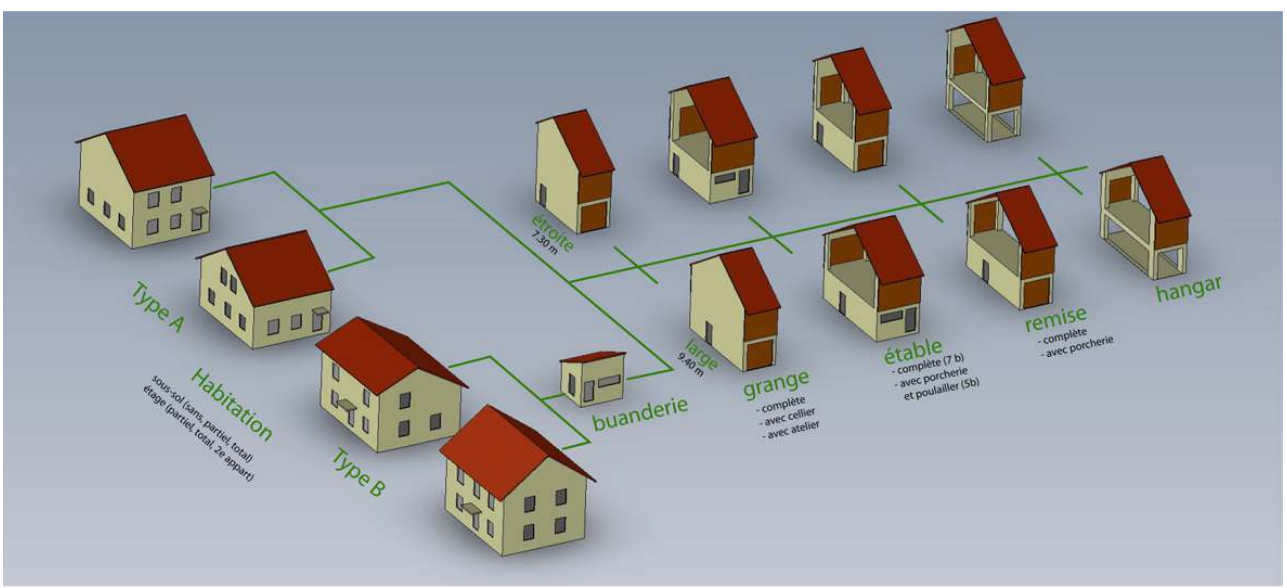

DESSIN DES DIFFÉRENTS CORPS DE BÂTIMENTS POUVANT COMPOSER UNE FERME DE L'OP 24.

Dessin Henry, Jean-Yves. Base Mémoire : IVR41_20128812160NUDA. @ Région Lorraine-Inventaire général. 


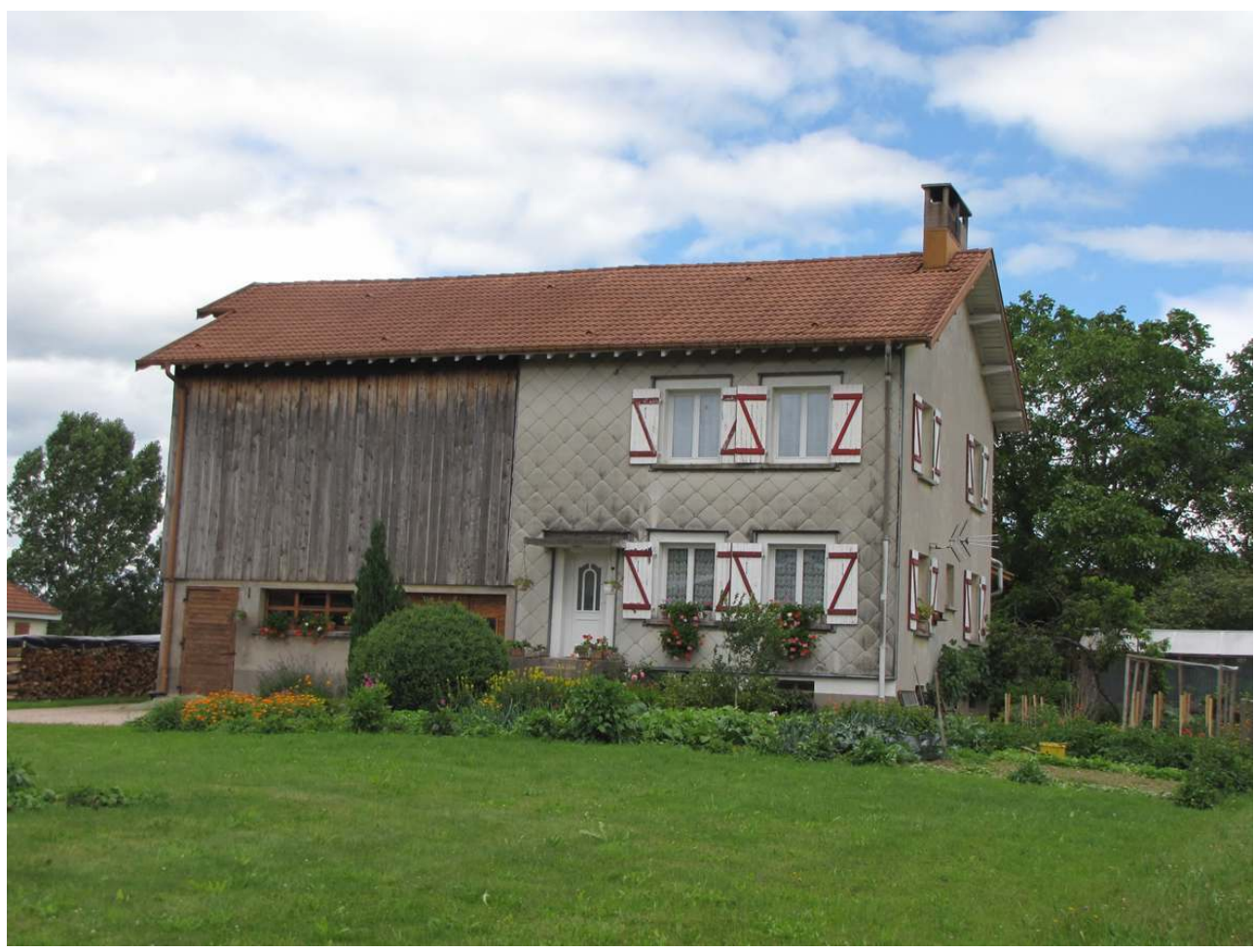

FERME DE L'OP 22 (HABITATION DE TYPE A2 AVEC gRANge ET ÉCURIE PROFONDE) À SAINT-LÉONARD.

Phot. Henry, Jean-Yves. Base Mémoire : IVR41_20128803347NUC2A. @ Région Lorraine-Inventaire général.

L'OP 24 propose une gamme de modules dans laquelle le sinistré puise pour composer sa ferme (fig. $\mathbf{n}^{\circ} \mathbf{2 3}$ ). L'habitation de type A associée à une exploitation permet de reconstituer un bâtiment au plan rectangulaire classique (fig. $\mathbf{n}^{\mathbf{0}} \mathbf{2 4}$ ). L'habitation de type $\mathrm{B}$ est reliée à l'exploitation par la buanderie qui forme un corps de passage (fig. $\mathbf{n}^{\circ}$ 25). L'association d'une habitation de type A à étage partiel avec une grange peu profonde et en retrait donne au bâtiment une allure surprenante (fig. $\mathbf{n}^{\circ} \mathbf{2 6}$ ). La première tranche porte sur 60 bâtiments. Ce plan sera à nouveau proposé pour l'OP 26. 
Figure 25

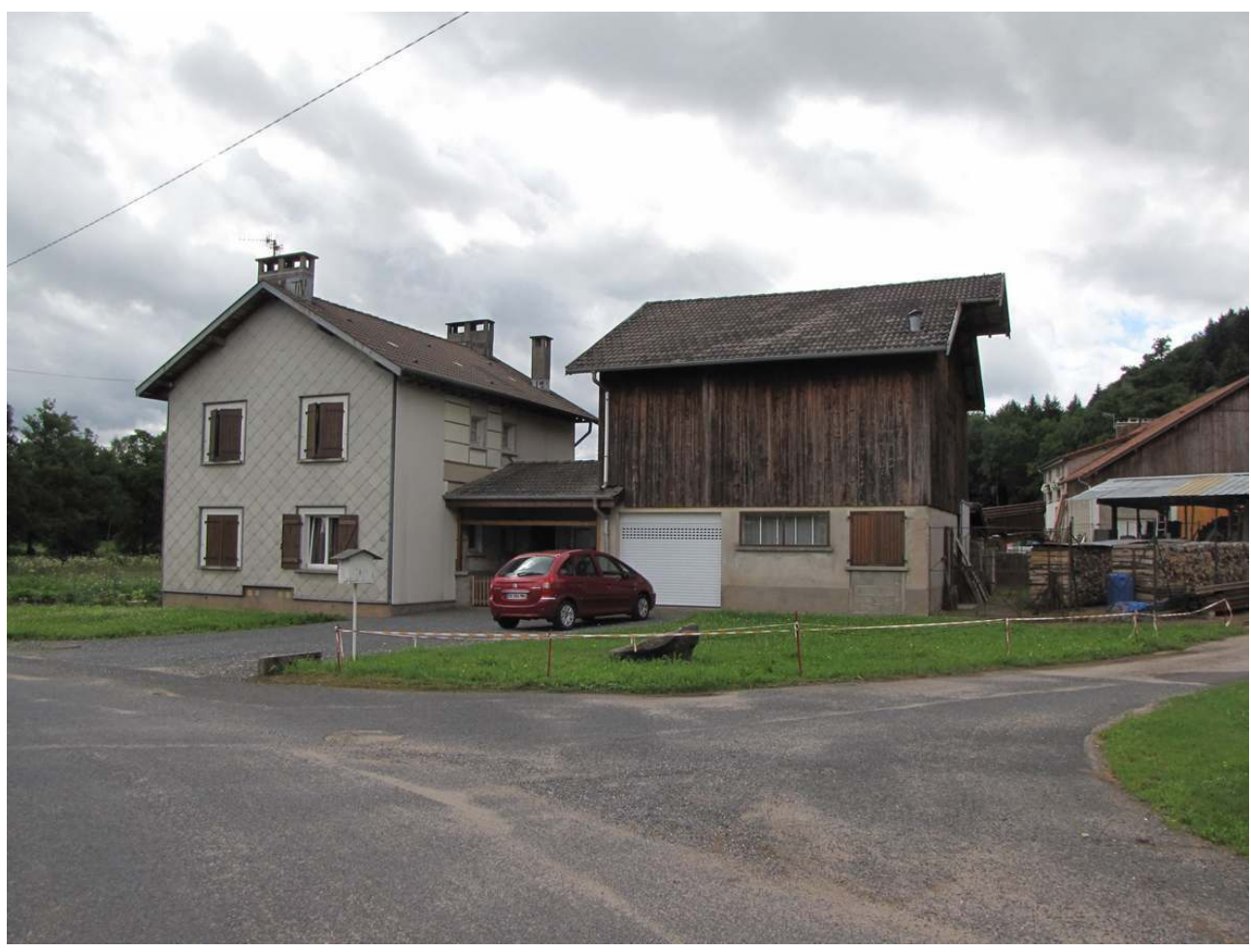

FERME dE L'OP 22 (HABITATION DE TYPE B2 AVEC CORPS DE PASSAgE, gRANgE ET ÉCURIE PROFONDE) À ANOULD.

Phot. Henry, Jean-Yves. Base Mémoire : IVR41_20128803349NUC2A. @ Région Lorraine-Inventaire général. 
Figure 26

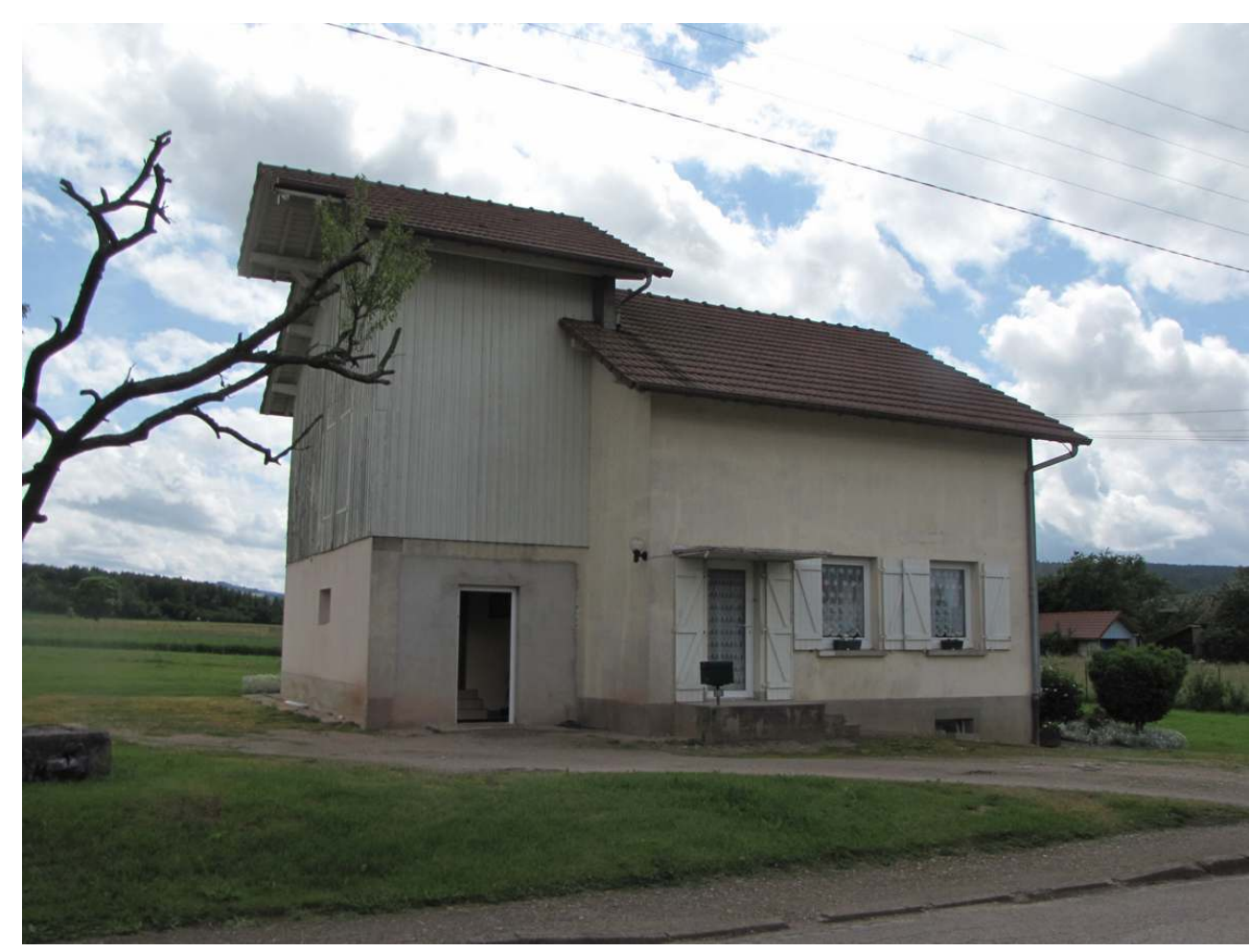

FERME de L'OP 22 (HABITATION DE TYPE A1 AVEC gRANgE PEU PROFONDE EN RETRAIT) À ANOULD.

Phot. Henry, Jean-Yves. Base Mémoire : IVR41_20128803348NUC2A. C) Région Lorraine-Inventaire général.

\section{Au-delà des opérations préfinancées}

40 Le dépouillement systématique des dossiers d'origine "agricole » sur les communes sélectionnées permet de constater la variété des modèles proposés. Si le «chalet suisse » reste une référence, de nombreux modèles atypiques sont construits (fig. $\mathbf{n}^{\circ} \mathbf{2 7}$ ). 


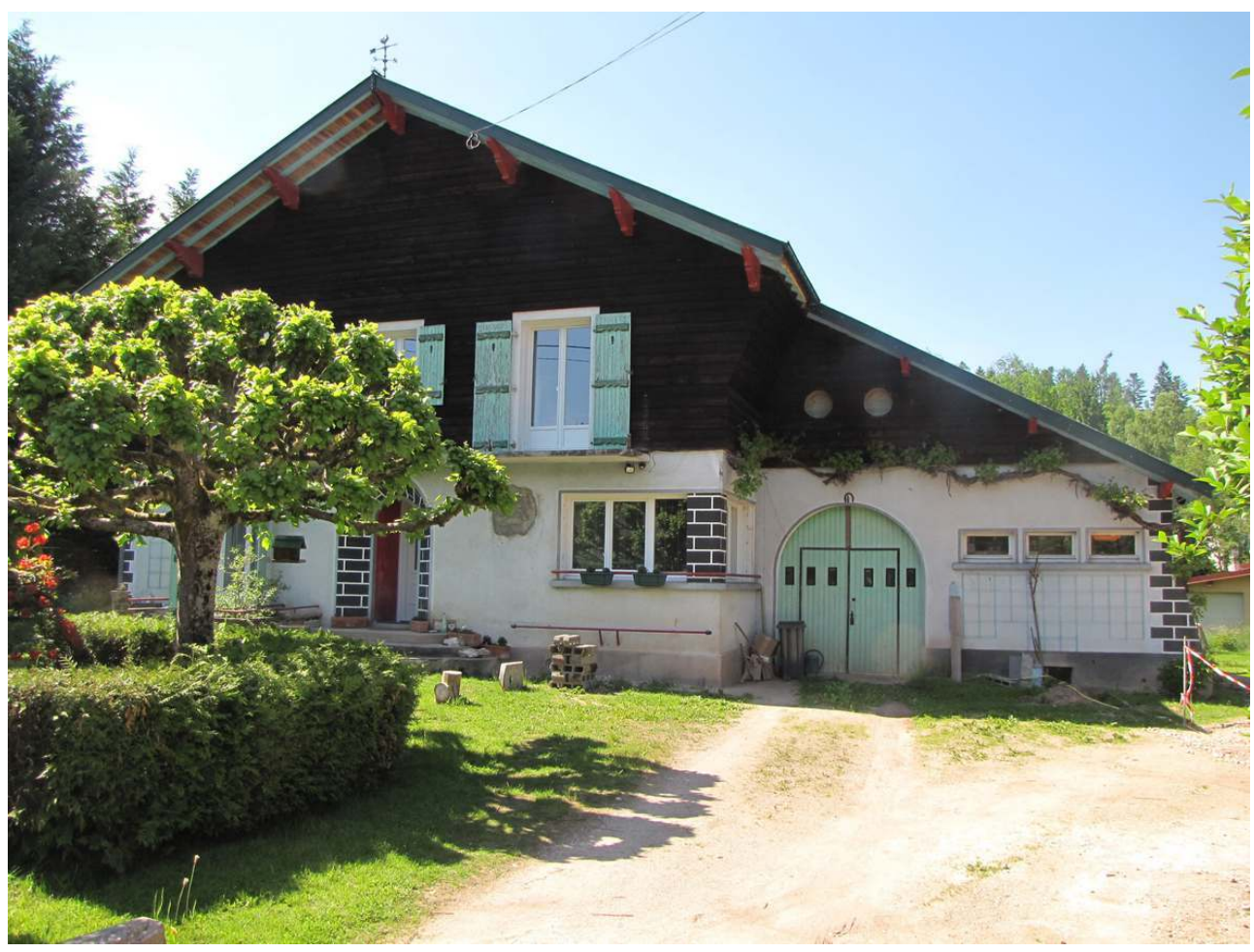

FERME CONSTRUITE SUR LES PLANS de L'ARChITECTE PIERRE PIAget À SAINT-LÉONARD.

Phot. Henry, Jean-Yves. Base Mémoire : IVR41_20128803355NUC2A. C Région Lorraine-Inventaire général.

41 Ce dépouillement permet aussi de constater que les mutations dans les destinations des bâtiments sont très faibles. Rares sont les édifices qui substituent à une fonction agricole une fonction purement résidentielle. En plein centre de La Bresse, on continue à construire des fermes ou des immeubles avec une partie agricole (basse-cour en rez-dechaussée, fenil et bûcher à l'étage). Cette dernière reste le plus souvent accolée au bâtiment principal, elle n'est qu'exceptionnellement rejetée en fond de cour dans une annexe. 


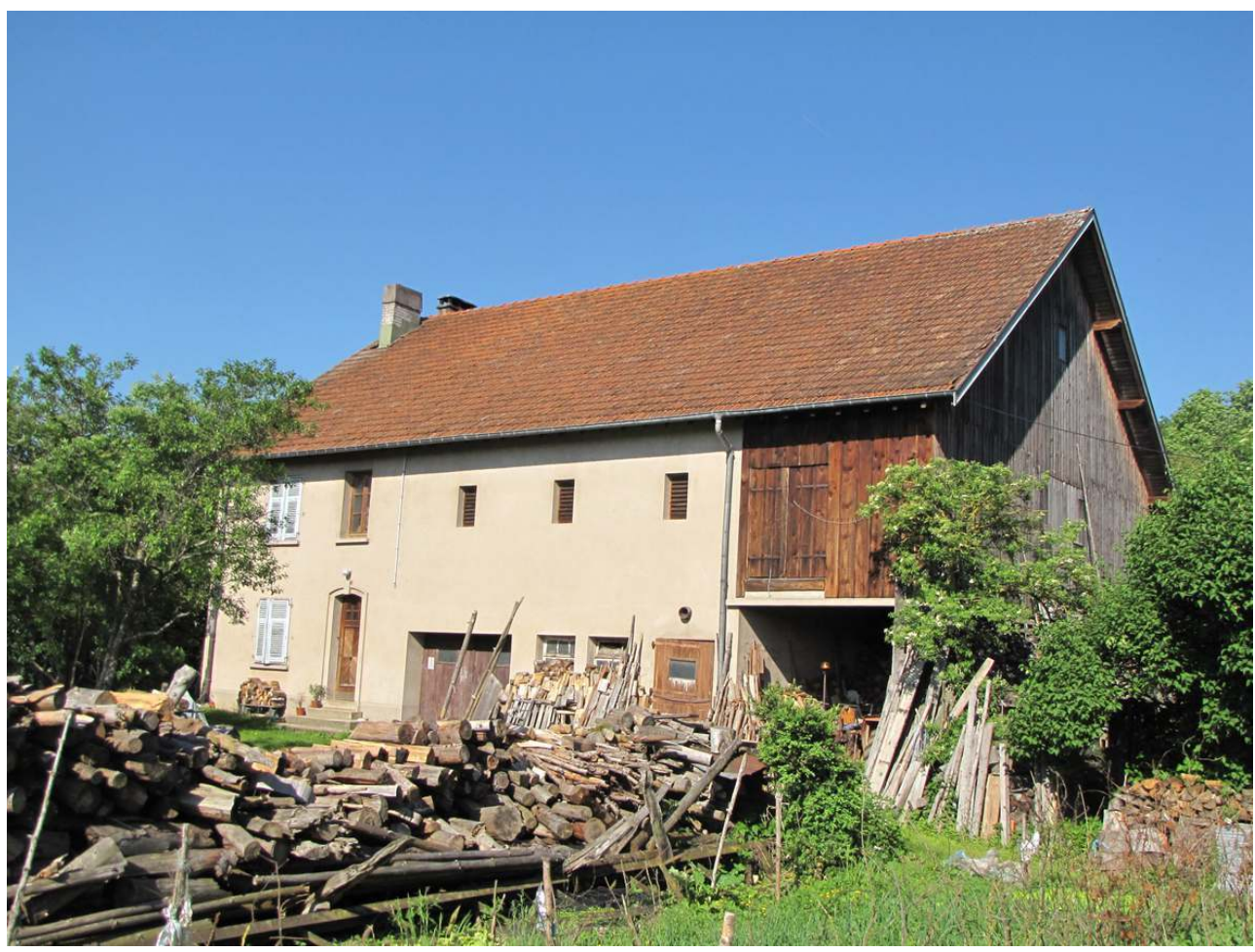

FERME DE L'OP 06 ENCORE EN ACTIVITÉ À SAINT-LÉONARD.

Phot. Henry, Jean-Yves. Base Mémoire : IVR41_20128803350NUC2A. C Région Lorraine-Inventaire général.

La nécessité de garder une activité rurale qui assure un complément de revenu est encore une évidence dans les années 1950. Si cette Seconde Reconstruction a su anticiper les aménagements urbains, elle n'a pas su entrevoir la profonde mutation du monde agricole survenue postérieurement. Aujourd'hui, la ferme standard conçue pour quelques bovins (cinq ou sept en règle générale et 22 au maximum comme à la ferme modèle de Saulcysur-Meurthe) a perdu, à quelques exceptions près (fig. $\mathbf{n}^{\circ} \mathbf{2 8}$ ), sa fonction agricole, mais les espaces réservés à l'exploitation, suffisamment modulables, ont su trouver une seconde destination. Après le remplacement des cloisons extérieures en bois par des murs en dur percés de baies (fig. $\mathbf{n}^{\circ} \mathbf{2 9}$ ), elles sont souvent transformées en habitation (second appartement), atelier ou garage. La majorité des fermes reconstruites ont aujourd'hui une fonction uniquement résidentielle (principale ou secondaire), sans avoir été gravement défigurées. 


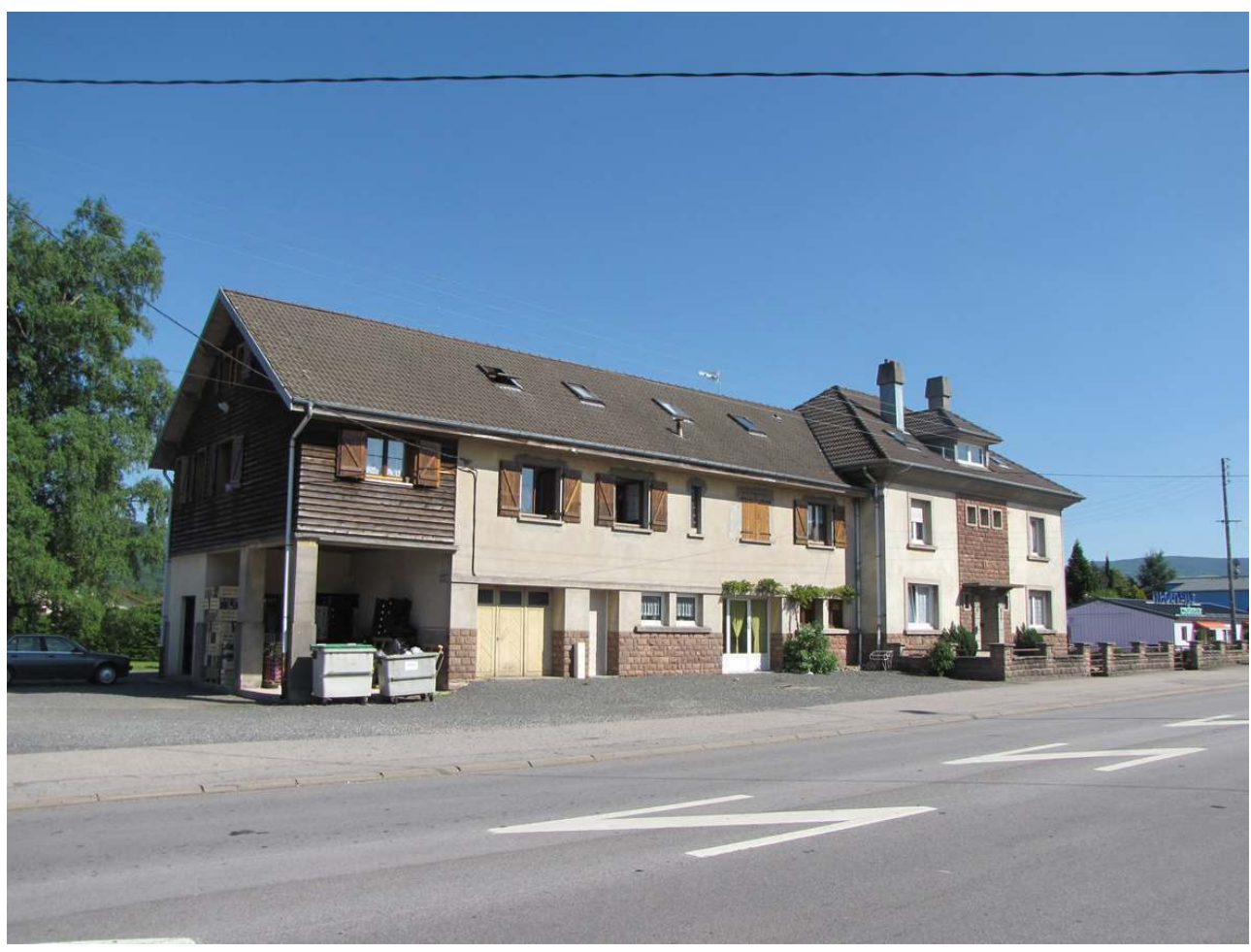

GRANDE FERME À DEUX LOgEMENTS DONT LES PLANS ONT ÉTÉ DRESSÉS PAR L'ARCHITECTE ROgER RINGWALD À SAINT-LÉONARD ET DONT LES DEUX NIVEAUX DE L'EXPLOITATION ONT ÉTÉ TRANSFORMÉS EN LOgEMENTS.

Phot. Henry, Jean-Yves. Base Mémoire : IVR41_20128803351NUC2A. @ Région Lorraine-Inventaire général.

\section{BIBLIOGRAPHIE}

VOLDMAN, Danièle. La reconstruction des villes françaises de 1940 à 1954 : histoire d'une politique. Paris : L'Harmattan, 1997. 487 p.

« Les Reconstructions des années 1920 et 1950 en Lorraine : un renouveau architectural et urbain ». La Gazette lorraine, 2011, hors-série, 111 p.

«Juvisy-sur-Orge. Un territoire, des réseaux » / Inventaire général du patrimoine culturel, Région Île-de-France. Lyon : Lieux Dits, 2007. - 264 p. : ill., plans ; 27 cm. - (Cahiers du patrimoine ;88).

ZEHLES, Thierry. « Un exemple d'opérations groupées de reconstruction : le quartier reconstruit du Boudiou à Épinal ». Annales de la société d'émulation du département des Vosges, 1985, p. 95-100.

[Exposition. Paris, Archives nationales. 1991]. « Reconstructions et modernisation. La France après les ruines 1918... 1945... : catalogue / exposition. Paris, Archives nationales. 1991 ». Paris : Archives nationales, 1991. 310 p., ill. 
[Exposition. Épinal, Espace Charles-de-Gaulle. 1991]. « Reconstruction et modernisation. Les Vosges après les ruines : catalogue / exposition. Épinal, Espace Charles-de-Gaulle. 1991 ». Épinal : conseil général des Vosges, 1991. 36 p.

DOYEN, Jean-Pierre. « Du chaos à la croissance : reconstruction et modernisation des HautesVosges de 1944 à 1960 ». Le pays de Remiremont. Actes des Journées d'études vosgiennes (2-4 novembre 2000 ; Remiremont), 2001, nº 15, p. 327-344.

\section{NOTES}

1. - L'ensemble des notices concernant la seconde reconstruction en milieu rural dans les Vosges sera versé dans la base Mérimée (IA88001853 à IA88001875...) dès la fin de l'étude.

2. - Chiffres donnés par le regroupement des ASR des Vosges dans ses rapports de 1949 et 1952. Arch. dép. Vosges 1815w1965 et 1966;

3. - Pierre Crouzillard (1906-1983), plus connu sous son prénom d'usage Jean, pour l'arrondissement d'Épinal et Georges Michau pour celui de Saint-Dié ; ils se partagent celui de Remiremont. Comme la majorité des architectes en chef du MRU, ils ont un cabinet à Paris.

4. - Entre 1946 et 1952, 134 architectes ont été agréés par le MRU pour le département des Vosges, ils sont classés en trois catégories (A : tous travaux dont édifices publics très importants ; $B$ : édifices publics de moyenne importance ; $C$ : réparation d'édifices publics). Parmi ceux-ci, 30 ont un cabinet en Région parisienne, mais en 1952, seulement 65 ont une activité suffisante pour faire l'objet d'une notation chiffrée par l'administration du MRU.

5. - Le sinistré choisit librement l'emplacement de la reconstruction, elle peut se faire dans une commune voisine. Pour rebâtir dans une autre région ou changer la destination du bâtiment, il doit bénéficier d'une dérogation.

6. - Nom donné dans l'habitat traditionnel lorrain à « la pièce de devant » chauffée au travers de la plaque de cheminée, servant de chambre des parents mais aussi de pièce de réception.

7. - OP 06, communes de Gerbépal et de Saint-Léonard, architecte: Roger Ringwald, deux variantes, 20 fermes construites, adjudication des travaux 1950. OP 10, communes d'Anould et de Ban-sur-Meurthe-Clefcy, trois groupes d'architectes : Albert Bourmeix, Jean-Claude Parvé, Lucien Toussaint- Michel Trefcon qui proposent chacun leur modèle en deux variantes, 30 fermes construites, adjudication des travaux 1951. OP15, Bru-Jeanménil, architecte : Pierre de Bois-Lucy, 10 fermes construites. OP 16, Rehaincourt, architecte : Louis Poisson, 12 fermes construites. OP 22 et 22bis, La Bresse, modèle 1 ou 3, architectes: Henri Gaillemin- Louis Mathieu, 35 fermes construites sur deux ans (1952-55). OP 24 et 24 bis, Saint-Dié-Fraize, un groupement d'architecte : Lucien Toussaint-Michel Trefcon pour la partie habitation, Francis Massé pour l'exploitation, Roger Ringwald-Roger Perrin pour les devis, 1 seul modèle dans de multiples variantes, 60 fermes construites, 1953. OP 26, Saint-Dié et Etival-Clairfontaine, architecte: Francis Massé, 14 bâtiments construits. OP 35, canton de Gérardmer, architectes : Igor Ivanov et Paul Poisson, 29 bâtiments construits.

8. - Charri : dans la partie sud de la montagne vosgienne, nom donné à la partie antérieure de la grange ; espace central qui dessert les différentes travées fonctionnelles de la ferme.

9. - Le coût moyen d'une ferme vosgienne reconstruite dans le cadre de l'OP 10 (la plus avantageuse) est estimé à 6400000 francs au 31 décembre 1951 pour une surface hors-œuvre pondérée de $450 \mathrm{~m}^{2}$. 


\section{RÉSUMÉS}

La montagne vosgienne a été durement touchée durant le second conflit mondial et certains villages totalement détruits. Face à l'abondance des archives du ministère de la Reconstruction et de l'Urbanisme (MRU) conservées aux archives départementales des Vosges, nous avons privilégié une approche fondée sur l'échantillonnage en sélectionnant deux zones à l'habitat traditionnel typologiquement différent, situées respectivement au nord et au sud de la montagne vosgienne, le val de Meurthe et La Bresse. C'est sur ces deux territoires qu'a été menée une étude plus approfondie. Les archives consultées nous permettent d'appréhender l'organisation administrative de la Reconstruction et d'analyser la recomposition urbaine des villes et villages détruits. Les dossiers d'indemnisation comportent les plans des bâtiments détruits et ceux des fermes reconstruites. Ils nous renseignent sur le choix des techniques et des matériaux employés en lien avec l'application des règles d'hygiène et de confort en vigueur. L'étude des dossiers des différentes opérations préfinancées d'immeubles ruraux permet d'esquisser les grandes tendances des partis de plans proposés par les nombreux architectes, aux niveaux technique, économique et esthétique. C'est aussi l'occasion de voir comment évoluent les modèles proposés sous l'influence des réflexions nationales ( $4^{\mathrm{e}}$ Congrès national de l'habitat rural), des directives des architectes en chef du ministère de la Reconstruction et de l'Urbanisme, des apports des architectes d'opération et également des choix des sinistrés.

The Vosges was severely affected during the Second World War and some villages were totally destroyed. Given the abundance of Ministry for Reconstruction and Town Planning (MRU) archives kept in the Vosges Departmental Archives, we gave priority to an approach based on sampling by selecting two typologically different areas of traditional habitat, situated respectively in the north and in the south of the Vosges Mountains; Val de Meurthe and La Bresse. It is in these two territories that a more comprehensive study was carried out. The archives consulted allow us to understand the administrative organization of the reconstruction and to analyse the rebuilding of the towns and villages which were destroyed. The compensation files include the plans both of buildings that were destroyed and the farms that were reconstructed. They give us information on the choice of techniques and the materials used in conjunction with the applicable regulations for hygiene and comfort. The study of the files of the different prefinanced operations for rural buildings allows us to outline the major trends in the sets of plans put forward by numerous architects on a technical, economical and aesthetic level. It is also an opportunity to see the evolution of the models offered in the light of national studies ( $4^{\text {th }}$ National Congress on the Rural Habitat), the directives issued by the head architects of the Ministry for Reconstruction and Town Planning, the contributions made by the on-site architects as well as the choice of disaster victims.

\section{AUTEUR}

\section{JEAN-YVES HENRY}

Chercheur au service de l'Inventaire général-Région Lorraine Jean-yves.henry@lorraine.eu 